\title{
Arabidopsis mlo3 mutant plants exhibit spontaneous callose deposition and signs of early leaf senescence
}

\author{
Stefan Kusch ${ }^{1}$, Susanne Thiery ${ }^{1}$, Anja Reinstädler ${ }^{1}$, Katrin Gruner $^{1}$, Krzysztof Zienkiewicz $^{2,3}$, Ivo Feussner ${ }^{2,3}$, and Ralph \\ Panstruga $a^{1, \square}$ \\ ${ }^{1}$ Unit of Plant Molecular Cell Biology, Institute for Biology I, RWTH Aachen University, Worringerweg 1, D-52056 Aachen, Germany \\ ${ }^{2}$ Department of Plant Biochemistry, Albrecht-von-Haller-Institute for Plant Sciences, Göttingen Center for Molecular Biosciences (GZMB), University of Göttingen, \\ Justus-von-Liebig-Weg 11, D-37077 Göttingen, Germany \\ ${ }^{3}$ Service Unit for Metabolomics and Lipidomics, Göttingen Center for Molecular Biosciences (GZMB), University of Göttingen, Justus-von-Liebig-Weg 11, D-37077 \\ Göttingen, Germany
}

\begin{abstract}
The family of Mildew resistance Locus $O$ (MLO) proteins is best known for its profound effect on the outcome of powdery mildew infections: when the appropriate MLO protein is absent, the plant is fully resistant to otherwise virulent powdery mildew fungi. However, most members of the MLO protein family remain functionally unexplored. Here, we investigate Arabidopsis thaliana MLO3, the closest relative of AtMLO2, AtMLO6 and AtMLO12, which are the Arabidopsis $M L O$ genes implicated in the powdery mildew interaction. The co-expression network of AtMLO3 suggests association of the gene with plant defenserelated processes such as salicylic acid homeostasis. Our extensive analysis shows that mlo3 mutants are unaffected regarding their infection phenotype upon challenge with the powdery mildew fungi Golovinomyces orontii and Erysiphe pisi, the oomycete Hyaloperonospora arabidopsidis, and the bacterial pathogen Pseudomonas syringae (the latter both in terms of basal and systemic acquired resistance), indicating that the protein does not play a major role in the response to any of these pathogens. However, mlo3 genotypes display spontaneous callose deposition as well as signs of early senescence in six- or seven-week-old rosette leaves in the absence of any pathogen challenge, a phenotype that is reminiscent of mlo $2 \mathrm{mu}$ tant plants. We hypothesize that de-regulated callose deposition in mlo3 genotypes is the result of a subtle transient aberration of salicylic acid-jasmonic acid homeostasis during development.
\end{abstract}

MLO (Mildew resistance Locus O) | salicylic acid (SA) | callose | senescence | phytohormone | powdery mildew | systemic acquired resistance (SAR)

Correspondence:

panstruga@bio1.rwth-aachen.de

\section{Abbreviations}

bp, base pair | CFU, colony forming units | dpi, days post inoculation | GO, gene ontology | GUS, $\beta$-glucuronidase | Hpa, Hyaloperonospra arabidopsidis | hpi, hours post inoculation | JA, jasmonic acid | JA-lle, jasmonic acid isoleucine | MAMP, microbe-associated molecular pattern | MS, Murashige and Skoog | Psm, Pseudomonas syringae pv. maculicola | Pst, Pseudomonas syringae pv. tomato | RH, relative humidity | RLU, relative light units | RT-PCR, reverse transcriptase polymerase chain reaction |SA, salicylic acid |SAG, salicylic acid glucoside | SAR, systemic acquired resistance | T-DNA, TransferDNA | UPLC-nano ESI-MS/MS, Ultrahigh-Pressure Liquid ChromatographyTandem Mass Spectrometry | YFP, yellow fluorescent protein

\section{Introduction}

Members of the family of Mildew resistance Locus O (MLO) proteins are well known for their intriguing effect regarding the modulation of susceptibility to powdery mildew fungi in plants. Loss-of-function mutants of the appropriate $M L O$ gene(s) confer durable broad-spectrum resistance against powdery mildews (1), an effect originally discovered in barley (2) and later validated in many monocotyledonous and dicotyledonous plant species (3).

In Arabidopsis, three genes (AtMLO2, At1g11310; AtMLO6, At1g61560; and AtMLO12, At2g39200) unequally contribute to susceptibility to powdery mildews. The mlo2 loss-offunction mutation alone causes macroscopically discernable resistance to the powdery mildew species Golovinomyces cichoracearum and G. orontii, but retains around 30-50\% entry rate by these fungi (4). Both mlo6 and mlo1 2 mutations potentiate the resistance phenotype of mlo2 mutants, but do not confer enhanced resistance to powdery mildew on their own. Only the triple mutant mlo 2 mlo6 mlo 12 is fully resistant, with $<1 \%$ entry success by the fungal invader (4). However, at least in barley and Arabidopsis the resistance trait is associated with pleiotropic phenotypes. Both the barley $m l o$ and the Arabidopsis mlo2 mutant exhibit spontaneous deposition of distinct callose aggregations in ageing but otherwise pathogen-free leaves (5), as well as early leaf senescence. The latter is first exemplified by mesophyll cell death (6) and later by catabolism of photosynthetic pigments, leaf chlorosis and necrosis $(4,5,7,8)$. Furthermore, the barley mlo and Arabidopsis mlo2 mlo6 mlo12 triple mutants are altered in the infection phenotype to a range of microbial phytopathogens unrelated to powdery mildews $(9,10)$. The Arabidopsis mlo2 single mutant also lacks systemic acquired resistance (SAR) against the bacterial pathogen Pseudomonas syringae (11) and exhibits enhanced tolerance to ozone (12). The barley Mlo gene codes for a seven-transmembrane domain protein with an extracellular or luminal amino-terminus and a cytosolic carboxy-terminus $(13,14)$. The carboxyterminal tail contains an evolutionarily conserved calmodulin binding site that is required for full functionality of barley Mlo towards barley powdery mildew infection (15). In addi- 
tion, four conserved cysteine residues in the first and third extracellular loop are required for barley Mlo function in the context of the powdery mildew interaction (16), and the short tetrapeptide motif [DE]FSF at the carboxy-terminus is a characteristic feature of MLO proteins involved in powdery mildew susceptibility (17). Several additional peptide motifs have been found that are highly conserved throughout MLO proteins of land plants $(18,19)$.

Comprehensive phylogenetic analyses of the MLO protein family have shown that MLO proteins can be grouped in at least seven distinct clades (19-21). All MLO proteins known to play a role in the powdery mildew interaction belong to clade IV in monocotyledonous plants (e.g. barley Mlo) and clade V in dicotyledonous plants (such as At MLO2, AtMLO6, and AtMLO12). The Arabidopsis genome encodes 15 MLO family members, represented by five of these clades (22). Apart from the interaction with powdery mildews, Arabidopsis MLO proteins have been implicated in root thigmomorphogenesis as the clade I mlo mutants mlo4 and mloll display a root curling phenotype upon a tactile stimulus in vitro $(23,24)$. The clade III mlo mutant nortia (nta, mlo7) causes reduced fertility since the female gametophyte is impaired in pollen tube recognition, resulting in pollen tube overgrowth in the synergids (25). AtNTA/AtMLO7 function requires localization to a Golgi-associated compartment, and the protein forms homo-oligomers during pollen tube perception (26).

AtMLO3 (At3g45290; clade VI) is closely related to AtMLO2, AtMLO6, and AtMLO12 (19), but a respective mlo3 knockout mutant did not display increased resistance to $G$. orontii at the macroscopic level (4). A $\beta$-glucuronidase (GUS) reporter construct under control of the AtMLO3 promoter revealed a similar expression pattern as a respective reporter construct driven by the AtMLO2 promoter, suggesting potentially overlapping or redundant function(s) of the two proteins (22). However, AtMLO3 and its encoded protein have never been functionally analyzed in detail. In this study, we expanded the analysis of the AtMLO3 expression profile and identified a set of co-expressed genes that overlap with those co-expressed with AtMLO2. We further characterized a set of mlo3 Transfer-DNA (T-DNA) insertion mutants with regard to the interaction with phytopathogens from various kingdoms of life. These comprised two powdery mildew fungi (the adapted species G. orontii and the nonadapted pea powdery mildew pathogen Erysiphe pisi), the oomycete Hyaloperonospora arabidopsidis (Hpa), and the bacterium $P$. syringae pv. tomato $(P s t)$. We further tested if, like in the case of mlo2, mlo3 mutants are impaired in SAR to $P$. syringae pv. maculicola (Psm). In addition, we analyzed whether mlo3 mutants, similar to mlo2 mutants, exhibit spontaneous callose deposition and early leaf senescence in unstressed conditions. Our data revealed no apparent role for AtMLO3 in plant immunity, but the findings suggest an overlapping function with AtMLO2 regarding the control of callose deposition and the appropriate onset of leaf senescence.

\section{Results}

Arabidopsis $M L O 3$ is co-expressed with defense response genes. AtMLO3 is phylogenetically the closest relative of AtMLO2, AtMLO6, and AtMLO12 (19), and according to promoter-GUS studies the four genes seem to be largely co-expressed (22). We used the electronic fluorescent pictograph (eFP) browser online tool (27) for a more detailed analysis of the AtMLO3 expression pattern. We noted high AtMLO3 transcript levels in the senescing leaf (Figure S1) and in the context of various biotic stresses. These comprise sites of $G$. orontii infection at 5 days post infection (dpi), challenge with the incompatible pathogens Pst DC3000 $\Delta h r c C$ and Hpa Emwa2 (in the Arabidopsis rpp4 mutant, which is susceptible to Emwa2), and treatment with the microbe-associated molecular patterns (MAMPs) flg22 and HrpZ. Weak AtMLO3 expression was further seen in the shoot during osmotic stress (Figure 1A). In addition, both AtMLO2, which was shown to exhibit a critical role during SAR (11), and AtMLO3 were up-regulated in systemic leaves upon primary Psm inoculation, as well as after irrigation with pipecolic acid in a FLAVIN MONOOXYGENASE1 (AtFMO1)-dependent manner (Table S1; data compiled from $(31,32))$. Pipecolic acid is a competent inducer of a SAR-like response and defense priming $(31,33)$. Together, the expression profile suggests an association of AtMLO3 function with leaf senescence, pathogen defense - in particular SAR, and/or possibly osmotic stress.

Next, we extracted the respective co-expression networks for AtMLO2, AtMLO3, and AtMLO1 (clade II MLO, which served as a negative control) using the ATTED-II online tool (28). Based on these data (Supplementary File 1) we performed gene ontology (GO) enrichment analysis with PLAZA 4.0 (29). The co-expression networks of AtMLO2 and AtMLO3 overlap by $26 \%$, i.e. 79 out of the 300 top co-expressed genes (Figure 1B). By contrast, there was little overlap between the co-expressed genes of AtMLO2 and AtMLO3, respectively, and those co-expressed with AtMLO1 (only 11 genes in total). Using PANTHER (release 2018_04; (30)), which provides a hierarchical GO term output, we analyzed the co-expression networks of AtMLO2, AtMLO3, and the 79 overlapping genes for over-representation of GO terms (Figure 1C, Table 1 and Supplementary File 1). Terms attributed to biotic stress response, incompatible interaction with pathogens, salicylic acid (SA) response, and SAR were highly represented in the co-expression networks of both genes. In case of AtMLO3 analyzed alone, SA biosynthesis and regulation were highly over-represented as well. Genes co-expressed with both AtMLO2 and AtMLO3 encode a number of proteins with well-defined functions in plant immunity (Supplementary File 1). For example, 20 genes (25\%) harbor the GO term "response to biotic stress", 27 genes (34\%) are annotated with "defense response", and eight genes are associated with "response to salicylic acid" (Supplementary File 2). The shared set comprises genes encoding a range of receptor-like kinases, including prominent members such as $A t$ BIR1 and $A t$ SOBIR1 (34), components of SA signaling (AtEDS1; (35)), transcriptional regulators of SA re- 


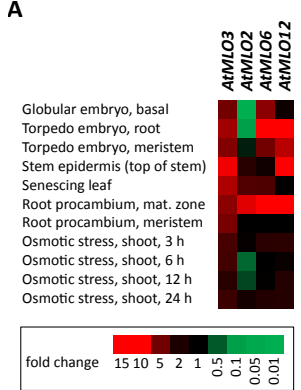

C

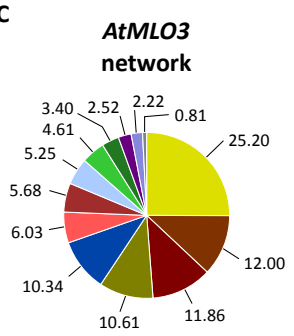

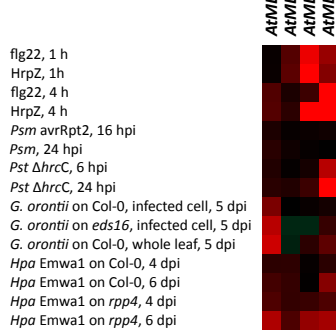
Hpa Emwa1 on rpp4, 6 dpi

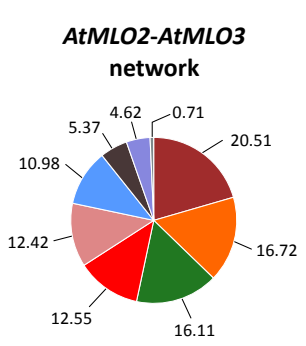

B

AtMLO1

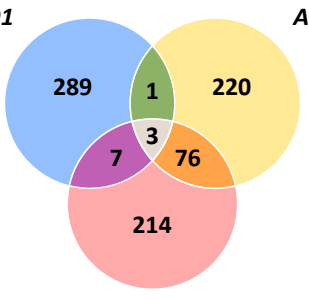

AtMLO2

regulation of SA biosynthetic process

regulation of SA-mediated signaling pathway response to $S A$

- SAR, SA mediated signaling pathway

positive regulation of response to biotic stimulus positive regulation of response to stimulus

positive regulation of defense response

response to molecule of bacterial origin

defense response to bacterium

defense response to bacterium, incompatible interaction

defense response, incompatible interaction

- defense response to fungus

regulation of innate immune response

regulation of response to external stimulus

- interspecies interaction between organisms

- plant-type hypersensitive response

protein phosphorylation

unclassified
AtMLO3

Fig. 1. AtMLO3 is co-expressed with components of plant immunity. A. Heatmap summarizing the relative expression in fold change of AtMLO2 (At1g11310), AtMLO3 (At3g45290), AtMLO6 (At1g61560), and AtMLO12 (At2g39200) upon the respective condition. Black, no change; red, increased expression level; green, decreased expression level. Data was extracted using the eFP browser (27). B. The top 300 co-expressed genes of AtMLO1 (At4g02600), AtMLO2, and AtMLO3 were extracted using ATTED-II release 2017.12.14 (28). The Venn diagram summarizes the number of overlapping genes. C. Venn diagrams highlighting enriched GeneOntology (GO) terms among the top 300 co-expressed genes of AtMLO3 (left) and among the 79 genes co-expressed in both AtMLO2 and AtMLO3 (middle). The legend to the right indicates the colors that are assigned to each term; the numbers indicate the percentage of genes in the respective network with that term. Gene enrichment analysis was done with PLAZA v4.0 (29) and GO terms were extracted using PANTHER v10 (30).
A
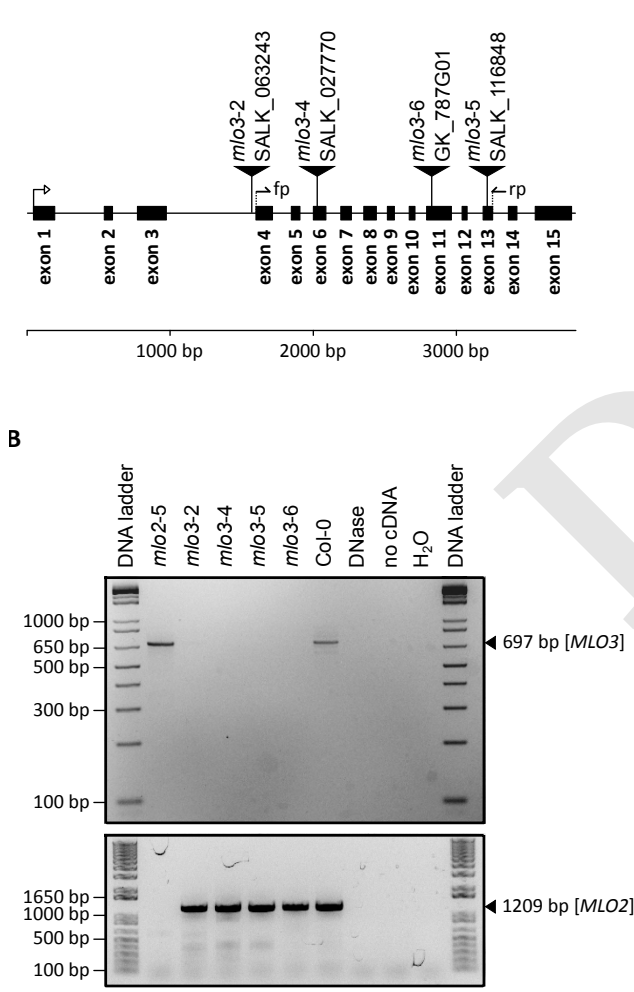

Fig. 2. Arabidopsis mlo3 T-DNA insertion lines do not express AtMLO3. A. Genomic map of AtMLO3. Black boxes represent exons, the lines in between introns. The location of the respective T-DNA insertions is indicated by triangles. $\mathrm{fp}$, forward primer (AtMLO_3C); rp, reverse primer (AtMLO_331). B. Upper panel, agarose gel for the RT PCR of the AtMLO3 transcript. The band is expected at 697 $\mathrm{bp}(\mathrm{fp}+\mathrm{rp})$, as indicated on the right. Lower panel, agarose gel for the RT-PCR of the AtMLO2 transcript (as control). The size of the band is $1,209 \mathrm{bp}$, as indicated on the right. Primers used are as described in (9). On the left, spacing of standard DNA fragments is indicated. DNA ladder, $1 \mathrm{~Kb}$ Plus DNA ladder (Invitrogen-Thermo Fisher, Waltham, MA, USA); DNase, water instead of RNA used for DNase digestion as template (negative control); no cDNA, water instead of RNA used for cDNA synthesis (negative control); $\mathrm{H}_{2} \mathrm{O}$, water template (negative control). sponses (AtNPR4; $(36,37)$ ), NBS-LRRs including AtRPS2 $(38,39)$, as well as some WRKY transcriptional regulators with known roles in plant defense and/or as negative regulators of plant senescence (AtWRKY53, AtWRKY54 and AtWRKY70; (40-42)).

We then assessed the 1000 base pair (bp) upstream regions of the 79 co-expressed genes from the common co-expression network of AtMLO2 and AtMLO3 for known and/or common cis-regulatory elements. Using the GO annotations from PLAZA4.0 (Supplementary File 2), we further sub-divided these genes into subgroups connected to defense or immune response, SAR, and SA-related processes. On the basis of analysis with AthaMap $(43,44)$, which performs databaseassisted searches for known cis-regulatory elements, we found that AtWRKY6 (five genes) and AtWRKY18 (seven genes) binding motifs were more than 4-fold overrepresented in the set of 79 genes (Supplementary File 3). Among the defense-related genes, AtWRKY6 was only found once, while other putative WRKY binding sites (AtWRKY12, AtWRKY18, AtWRKY38, and AtWRKY43) as well as two MYB factor binding sites (AtMYB.PH3, AtMYB98) occurred multiple times each. Next, we subjected the $1000 \mathrm{bp}$ upstream sequences of the 79 genes to an unbiased detection of sequence motifs using MEME v5.0.4 (45). While the query sequences derived from genes associated with the GO terms SA and SAR did not yield any significant motifs at $\mathrm{p}<1 \mathrm{e}-2$, two non-homopolymeric motifs were overrepresented in the upstream regions of genes sharing GO terms related to defense response (Supplementary File 4). Using the MEME-TOMTOM tool against the Arabidopsis thaliana DAP motif database, we found that these may represent AtWRKY47 and AtRAP2.12 binding sites. Overall, our cis-regulatory element analysis suggests that the co-expression network of AtMLO2 and AtMLO3 
Table 1. Highly over-represented biological process GO terms in the common co-expression network of AtMLO2 and AtMLO3.

\begin{tabular}{llll}
\hline GO-term & Log2-Enrichment & p-value & Description \\
\hline GO:1902478 & 6.31 & $8.21 \mathrm{E}-04$ & Negative regulation of defense response to bacterium, incompatible interaction \\
GO:0080142 & 6.16 & $2.80 \mathrm{E}-05$ & Regulation of salicylic acid biosynthetic process \\
GO:0009697 & 5.67 & $8.04 \mathrm{E}-05$ & Salicylic acid biosynthetic process \\
GO:0009862 & 5.31 & $1.74 \mathrm{E}-04$ & Systemic acquired resistance, salicylic acid mediated signaling pathway \\
GO:0010337 & 5.21 & $2.16 \mathrm{E}-04$ & Regulation of salicylic acid metabolic process \\
GO:0031098 & 5.21 & $2.16 \mathrm{E}-04$ & Stress-activated protein kinase signaling cascade \\
GO:0010112 & 5.11 & $2.65 \mathrm{E}-04$ & Regulation of systemic acquired resistance \\
GO:0046189 & 4.82 & $4.87 \mathrm{E}-04$ & Phenol-containing compound biosynthetic process \\
GO:0042391 & 4.78 & $5.26 \mathrm{E}-04$ & Regulation of membrane potential \\
GO:0009682 & 4.67 & $6.56 \mathrm{E}-04$ & Induced systemic resistance \\
GO:0009696 & 4.51 & $9.14 \mathrm{E}-04$ & Salicylic acid metabolic process \\
GO:0048544 & 4.35 & $1.32 \mathrm{E}-04$ & Recognition of pollen \\
GO:0009816 & 4.32 & $1.66 \mathrm{E}-06$ & Defense response to bacterium, incompatible interaction \\
GO:0008037 & 4.27 & $1.63 \mathrm{E}-04$ & Cell recognition \\
GO:0031348 & 4.26 & $1.88 \mathrm{E}-05$ & Negative regulation of defense response \\
GO:0009626 & 4.22 & $2.53 \mathrm{E}-06$ & Plant-type hypersensitive response \\
GO:0034050 & 4.21 & $2.66 \mathrm{E}-06$ & Host programmed cell death induced by symbiont \\
GO:0009875 & 4.21 & $1.91 \mathrm{E}-04$ & Pollen-pistil interaction \\
GO:0009627 & 4.17 & $2.54 \mathrm{E}-05$ & Systemic acquired resistance \\
\hline
\end{tabular}

might be mainly regulated by WRKY transcription factors, in particular those genes in this network related to the plant defense response.

We next used a transgenic promoter-GUS (PAtMLO3::GUS) line, and as a control a PAtMLO2::GUS line (22), to examine whether the expression pattern revealed by in silico analysis can be detected histologically upon ageing, pathogen stress, wounding, or MAMP treatment. As previously reported (7), strong GUS signals were seen in ageing rosette leaves of the PAtMLO2::GUS line; however, we found hardly any detectable GUS signal with the PAtMLO3::GUS line, not even in eight-week-old plants grown under long day conditions (Figure S2A). In case of the AtMLO3::GUS line, GUS staining was detectable in spots in old rosette leaves of plants grown under short day conditions, in trichomes, and occasionally in the meristematic zone of small leaves (Figure S2B). Similarly, only the PAtMLO2::GUS line was responsive to wounding by tweezers, with GUS signal detectable as late as $24 \mathrm{~h}$ after wounding, while the PAtMLO3::GUS plants showed no recognizable response (Figure S2C). To test the reaction of the two GUS reporter lines to MAMPs, we treated seedlings grown in liquid Murashige and Skoog (MS) medium with flg22 (100 nM) or chitin $\left(100 \mu \mathrm{g} \mathrm{mL}^{-1}\right)$ and found the PAtMLO2::GUS line to be somewhat responsive, while we observed no GUS signal in PAtMLO3::GUS seedlings (Figure S2D). Both transgenic promoter-GUS lines lacked a detectable increase in GUS staining upon challenge with virulent pathogens ( $G$. orontii, Hpa, or Pst DC3000), equally upon macroscopic inspection and following microscopic examination of infection sites (Figure S2E). Taken together, publicly available microarray and co-expression data suggests that AtMLO3 might be associated with senescence and biotic stress responses, while the transgenic promoter-GUS line did not support this notion.
Mutations in AtMLO3 do not affect compatible and incompatible powdery mildew interactions. Since At MLO3 is closely related to clade V MLO proteins like $A t \mathrm{MLO} 2, A t \mathrm{MLO}$, and $A t \mathrm{MLO} 12$, we hypothesized that AtMLO3 might also be involved in the modulation of powdery mildew interactions. We used four independent T-DNA insertion lines as single mutants (mlo3-2 (4), mlo3-4, mlo3-5 and mlo3-6) to test this possibility experimentally (Figure 2A). We failed to detect the full-length transcript of AtMLO3 in these lines by reverse transcription-polymerase chain reaction (RT-PCR) analysis, indicating that they are true knockout mutants (Figure 2B). Furthermore, we created double and higher order mutants of mlo3-4 in combination with mlo2-5, mlo6-2, and mlo12-1. When challenged with the adapted powdery mildew pathogen $G$. orontii, all four mlo3 single mutant lines showed similar susceptibility as the wild type accession Col-0, with about $80 \%$ entry success at 48 hours post inoculation (hpi) and intense sporulation at 7 dpi (Figure 3A and Figure S3). Similarly, the combinations of mlo3-4 with mlo2-5, mlo6-2, and mlo12-1 exhibited the same levels of penetration success at 48 hpi as the respective mutant line with wild type $M L O 3$ present (Figure $3 \mathrm{~B})$, and all plants harboring the mlo2-5 mutation were without macroscopically visible powdery mildew symptoms at 7 dpi (Figure S3). Next, we tested the interaction of mlo3 mutants with the non-adapted pea powdery mildew pathogen Erysiphe pisi. Upon challenge with this fungus, the mlo3 single mutants displayed similar entry rates of about $15 \%$ as the Col-0 wild type. The combination with mlo2-5 did not change the entry rate significantly (Figure 3C), suggesting that AtMLO3 also does not play a prominent role in defense against the non-adapted powdery mildew pathogen.

We further performed transient overexpression of AtMLO2 C-terminally tagged with yellow fluorescent protein (AtMLO2-YFP) and AtMLO3-YFP in Arabidopsis leaf epidermal cells to analyze comparatively the subcellular 

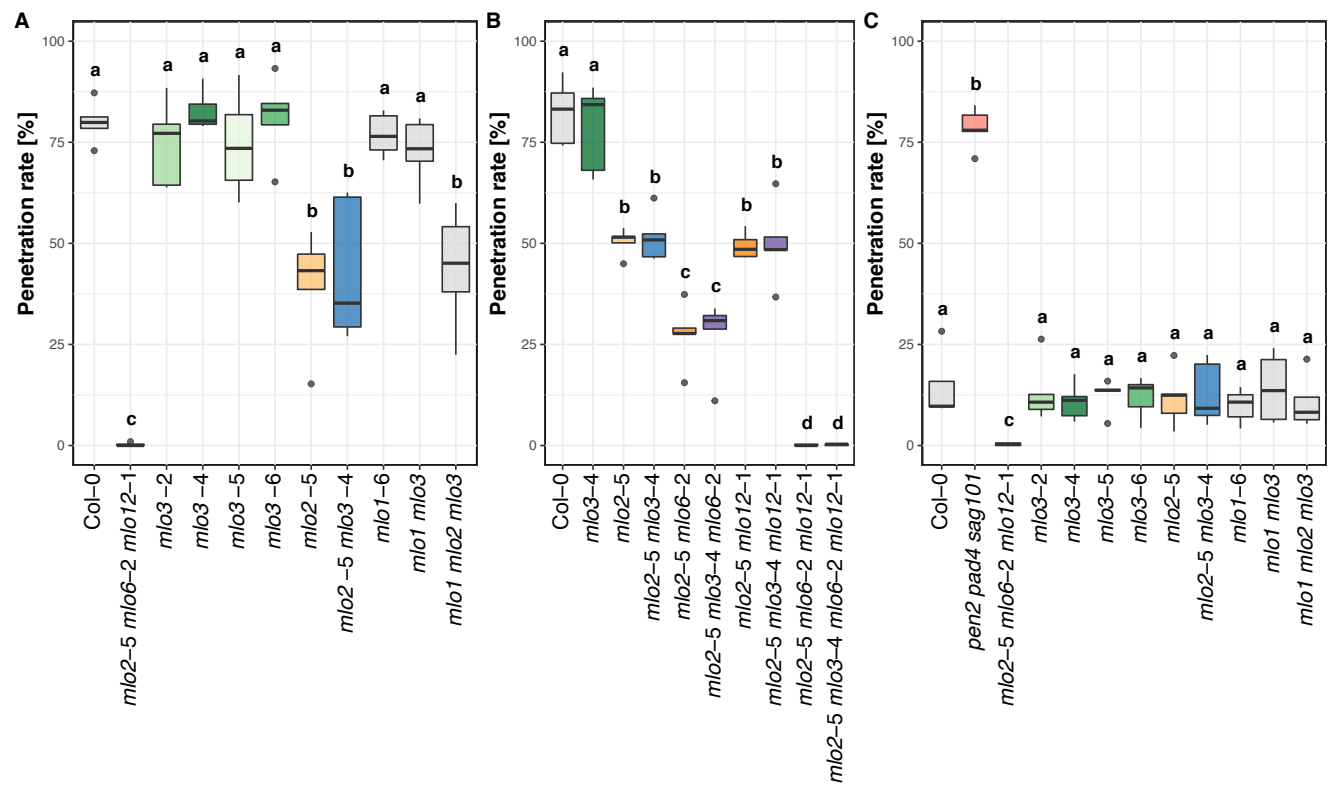

Fig. 3. Arabidopsis mlo3 mutants show unaltered powdery mildew infection phenotypes. Plants were inoculated with the indicated powdery mildew pathogen and the penetration rate was determined at $48 \mathrm{hpi}$. A. Boxplot showing G. orontii penetration rates for the mlo3 mutant lines (Figure $2 \mathrm{~A}$ ) and for mutant combinations with $\mathrm{mlo} 2-5$ and mlo1-6. Col-0 is the wild type, mlo2-5 mlo6-2 mlo12-1 the resistant control, mlo2-5 and m/o1-6 are parental controls for the mutant combinations. B. Boxplot showing penetration success of $G$. orontii for mutant combinations of mlo3-4 with mlo2-5, mlo6-2 and mlo12-1. Col-0 and mlo3-4 are susceptible controls, the respective parent control harboring wild type AtMLO3 is always placed left from the mlo3-4-containing mutant combination. C. Boxplot for the E. pisi penetration rate with the same mutants as in (A), including the hyper-susceptible control pen2-1 pad4-1 sag101-1 (46). Each replicate is based on 150-200 interaction sites scored on four to five leaves from one leaf per plant and genotype; data shown is based on the means from each replicate. $n=5$ independent replicates in all experiments. Statistical testing was done with GLM (binomial distribution).

localization of the two AtMLO proteins. AtMLO2-YFP localized to the cell periphery and was additionally present in discrete vesicular structures in the cells, while AtMLO3YFP seemed to reside exclusively at the cell periphery (Figure 4). We found that AtMLO2-YFP re-localized to and accumulated at penetration sites of $G$. orontii, while subcellular localization of AtMLO3-YFP was seemingly unaffected by the challenge with this pathogen (Figure 4). Altogether, our data indicates that AtMLO3 is not involved in the interaction between Arabidopsis and powdery mildew fungi.

\section{mlo3 mutants show wild type-like colonization by Hpa} and Pst. Due to the apparent association of AtMLO3 with components and processes of plant defense (Figure 1), we suspected that despite the fact that powdery mildew susceptibility was unaffected in mlo3 mutant plants, AtMLO3 might play a role in modulating defense to other pathogens. To test this idea, we employed the hemi-biotrophic bacterial pathogen Pst DC3000 and the biotrophic oomycete Hpa Noco 2 in our experiments. Hpa Noco2 (virulent on Col-0) was spray-inoculated and the reproductive success was determined as (conidio-)spores per $\mathrm{g}$ fresh weight at $7 \mathrm{dpi}$. We found that all tested genotypes (mlo3 single mutants, mlo1-6, mlo2-5, and combinations of mlo3-4 with mlo1-6 and/or mlo2-5) allowed oomycete sporulation at levels of 2.5$7.5 \times 10^{5}$ spores/g FW (Figure 5A), which is comparable to the Col-0 wild type. The Arabidopsis accession Landsberg erecta (L-er; harboring the Hpa resistance gene RPP5) was fully resistant and the eds1-2 [Col-0] mutant was more susceptible ( $c$. $10 \times 10^{5}$ spores/g FW) to Hpa Noco2, as pre-

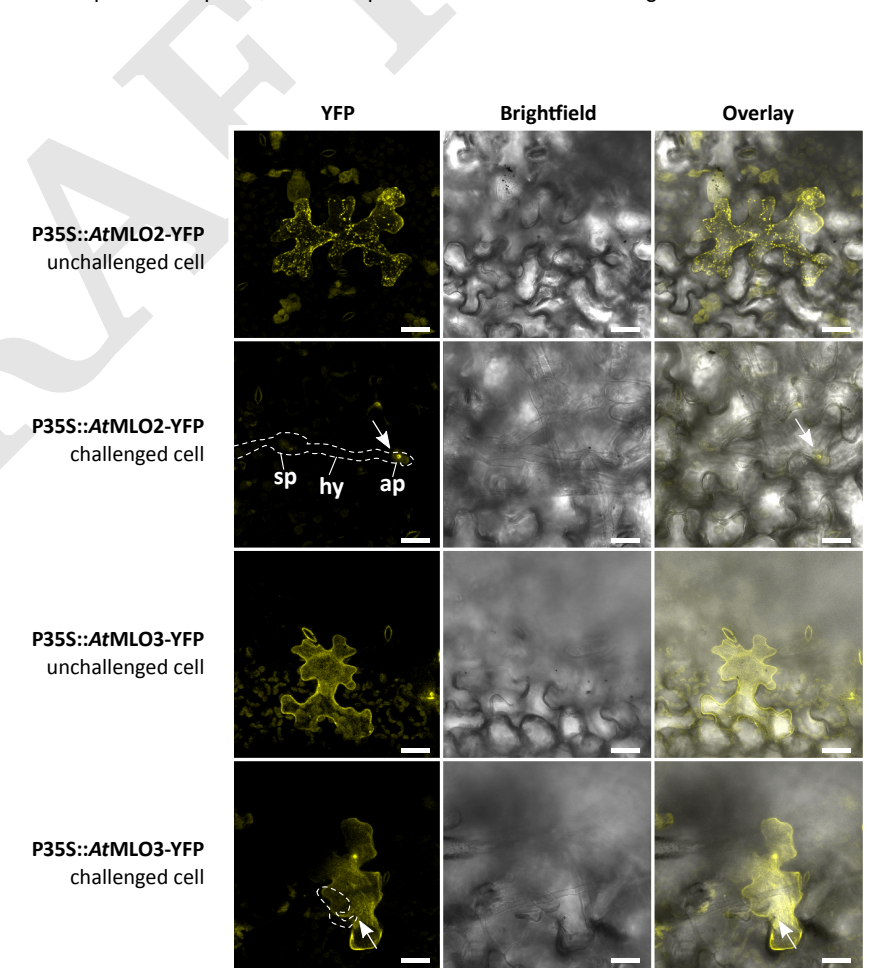

Fig. 4. AtMLO3-YFP does not re-localize to powdery mildew penetration sites. AtMLO2-YFP (upper two panels) and AtMLO3-YFP (lower two panels) were transiently over-expressed under control of the cauliflower mosaic virus $35 \mathrm{~S}$ promoter in leaf epidermal cells of Arabidopsis mlo2-5 mlo3-4 mlo6-2 mlo12-1 plants by use of biolistic gold particle delivery. "Challenged cell" designates cells challenged with G. orontii at $48 \mathrm{hpi}$ (fungal structures indicated by the dashed line). The left micrographs show the YFP signal (YFP excitation at $514 \mathrm{~nm}$, emission at 525-570 nm), the middle micrographs represent the respective brightfield image and the right micrographs depict the overlay of both. Confocal YFP images were generated by 3D reconstruction and are shown as maximum projection of all layers. Arrows point at the G. orontii penetration sites. sp, spore; ap, appressorium; hy, secondary hyphae. Scale bar: $25 \mu \mathrm{m}$. 

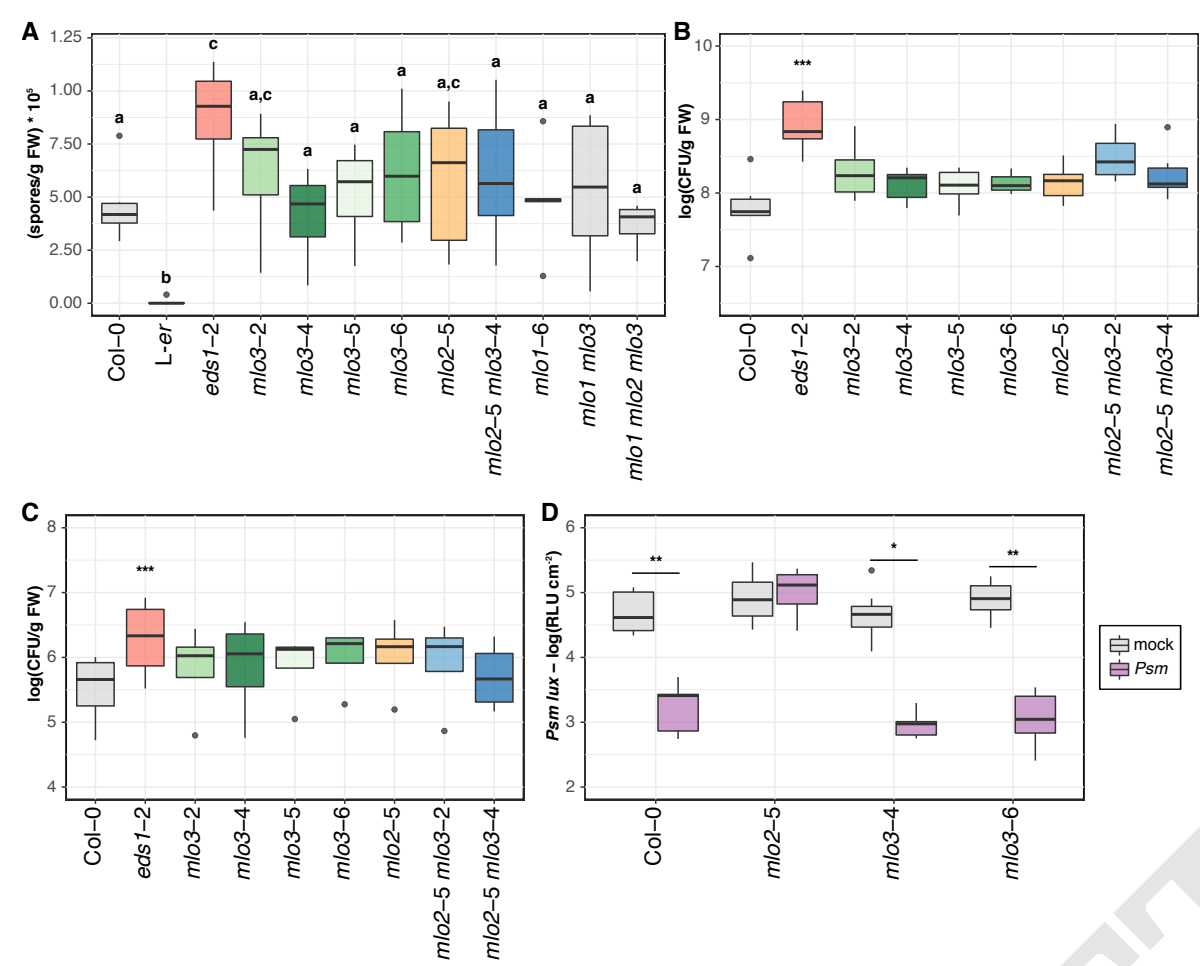

viously reported for these genotypes $(47,51)$. The result of this experiment indicates that neither $m l o 2$ nor $m l o 3$ mutants show an altered infection phenotype to the adapted oomycete Hpa Noco2.

Pst DC3000 (virulent) and its derived mutant strain Pst DC3000 $\Delta h r c C$ (lacking the type III secretion apparatus and being strongly compromised in virulence; (50)) were inoculated on 16-day-old Arabidopsis seedlings by sprayinoculation, and the bacterial titer in rosette leaves was determined as colony-forming units (CFU) at 0 dpi (inoculation control; see Figure S4) and at 3 dpi. The Col-0 wild type displayed a bacterial titer of $\mathrm{ca} .10^{8} \mathrm{CFU} / \mathrm{g} \mathrm{FW}$, while the hyper-susceptible control eds 1-2 [Col-0] (48) had a significantly enhanced titer of around $10^{9} \mathrm{CFU} / \mathrm{g} \mathrm{FW}$ (Figure 5B). The mlo3 and mlo2-5 single mutants as well as the mlo2-5 mlo3-2 and mlo2-5 mlo3-4 double mutants all showed a slightly increased bacterial titer compared to Col-0 $\left(>10^{8}\right.$ $\mathrm{CFU} / \mathrm{g}$ FW), but the observed differences were not significant in our set of experiments according to thorough statistical testing. Similarly, all these mutant lines revealed bacterial titers comparable to Col-0 following inoculation with Pst DC3000 $\Delta h r c C$ (around $10^{6} \mathrm{CFU} / \mathrm{g} \mathrm{FW}$ ); only eds $1-2$ [Col-0] was more susceptible with $\mathrm{ca}$. $10^{7} \mathrm{CFU} / \mathrm{g}$ FW (Figure 5C). Finally, since both publicly available microarray coexpression data (Table 1) and deep transcriptome sequencing (RNA-Seq) data (Table S1) indicate a possible functional association of AtMLO3 with SAR, we investigated the ability of mlo3 mutant lines to exhibit SAR against Psm. Three primary leaves of five-week-old plants were pressure-infiltrated with the virulent Psm strain ES4326, followed by inoculation of three systemic leaves with Psm lux (a transgenic variant of
Fig. 5. Arabidopsis mlo3 mutants show unaltered Hpa and Pst infection phenotypes and are SARcompetent in association with Psm. A. Hpa sporulation measured at $7 \mathrm{dpi}$ in (conido-)spores per gram fresh weight $(F W)$. Genotypes are as in Figure 3A, with Col-0 being the susceptible wild type, accession L-er the resistant control (47), and eds 1-2 in Col-0 background (48) the hyper-susceptible control. $n=6$ independent replicates, and GLM (Poisson distribution) was used for statistical testing. B. Pst DC3000 bacterial proliferation was determined at $3 \mathrm{dpi}$ in Arabidopsis seedlings after spray inoculation. The bacterial titer is expressed as log10 of colony-forming units (CFU) per gram fresh weight. Col-0 is the wild type, mlo2-5 a parental and eds1-2 a hyper-susceptible control. The four mlo3 mutant lines and the double mutants mlo2-5 mlo3-2 and mlo2-5 mlo3-4 were subjected to Pst inoculation. $\mathrm{n}=6$ independent replicates with GLM (Poisson distribution) as statistical test. C. Data of bacterial infection assay as in (B), with the avirulent strain Pst DC3000 $\Delta$ hrcC $(49,50)$. $\mathrm{n}=4$ independent replicates using GLM (Poisson distribution) as statistical test. D. SAR was monitored by primary infiltrations of three lower leaves of fiveweek-old plants with $P s m$ or $10 \mathrm{mM} \mathrm{MgCl}_{2}$ (mock), followed by inoculations of systemic leaves with Psm lux two days later. Bacterial proliferation of Psm lux was measured at $3 \mathrm{dpi}$, expressed as relative light units (RLU) $\mathrm{cm}^{-2} . \mathrm{n}=1$ biological replicate with nine plants per genotype, statistical testing was performed by GLM (quasi-Poisson distribution model). Similar results were obtained in a second independent experiment (Figure S4).
Psm constitutively expressing luciferase; (52)) two days later. Bacterial proliferation of Psm lux was quantified at 3 dpi by measuring luciferase activity (given as relative light units, RLU). Both mlo3 mutant lines were competent in acquiring resistance to Psm lux after pre-inoculation with Psm in a wild type-like manner, with approximately $10^{3} \mathrm{RLU} \mathrm{cm}^{-2}$ after pre-infection with $P s m$, compared to $c a$. $10^{5} \mathrm{RLU} \mathrm{cm}^{-2}$ after mock treatment (Figure 5D and Figure S4C). By contrast, the mlo2-5 mutant revealed similar RLU values upon both mock and Psm pre-treatment, since this mutant is SARincompetent (11). Together, these data suggest that Arabidopsis mlo3 mutants are not altered regarding colonization by the bacterial pathogen Pst DC3000 and they do not show a lack of SAR against Psm lux in our experimental conditions.

mlo3 mutants are not affected in development upon osmotic stress. Guided by the slightly induced expression levels of AtMLO3 in roots upon osmotic stress (Figure 1A), we determined the osmotic stress tolerance of mlo3 mutant lines in vitro. Seedlings grown on MS plates containing $300 \mathrm{mM}$ of mannose were quantitatively assessed for root length, the number of lateral roots, fresh weight, and leaf number (developmental stage). We found that with a few outliers, the mlo2 and mlo3 single mutants as well as the tested mlo2 mlo3 double mutants behaved like the wild type Col-0 in all respects. The root length of the two-week-old seedlings was in the range of $10-15 \mathrm{~mm}$ in our setting, as opposed to ca. $50 \mathrm{~mm}$ in non-stress conditions. The number of lateral roots was between one and two, the fresh weight was at 1$2 \mathrm{mg}$ per plant, and the leaf number, which was usually four when unstressed, was at least eight (Figure S5). Accordingly, 
bioRxiv preprint doi: https://doi.org/10.1101/558122; this version posted February 22, 2019. The copyright holder for this preprint (which was not certified by peer review) is the author/funder. All rights reserved. No reuse allowed without permission.

Table 2. Average and maximum callose deposition in rosette leaves of six-week-old plants.

\begin{tabular}{lllll}
\hline & Replicate 1 & & Replicate 2 & \\
\hline Genotype & Mean \pm SD & Maximum & Mean \pm SD & Maximum \\
\hline Col-0 & $6.3( \pm 3.8)$ & 16.3 & $3.4( \pm 1.7)$ & 7.6 \\
mlo3-2 & $25.6( \pm 46.2)$ & 266.3 & $6.4( \pm 7.5)$ & 37.4 \\
mlo3-2 pmr4-1 & $1.7( \pm 2.4)$ & 9.4 & $2.1( \pm 1.9)$ & 8.1 \\
mlo3-4 & $11.9( \pm 12.4)$ & 48.1 & $8.0( \pm 9.0)$ & 63.0 \\
mlo3-4 pmr4-1 & $3.7( \pm 4.1)$ & 16.0 & $2.7( \pm 1.9)$ & 8.5 \\
mlo2-5 & $59.0( \pm 48.6)$ & 224.3 & $12.8( \pm 15.5)$ & 78.9 \\
mlo2-5 mlo3-2 & $33.4( \pm 38.4)$ & 135.3 & $8.2( \pm 12.3)$ & 83.2 \\
mlo2-5 mlo3-4 & $69.2( \pm 53.0)$ & 206.4 & $40.7( \pm 41.5)$ & 174.1 \\
mlo2 mlo6 mlo12 & $99.8( \pm 87.5)$ & 297.2 & $11.7( \pm 18.0)$ & 85.9 \\
mlo2 mlo3 mlo6 mlo12 & $55.0( \pm 52.8)$ & 177.1 & $43.5( \pm 62.8)$ & 265.0 \\
\hline
\end{tabular}
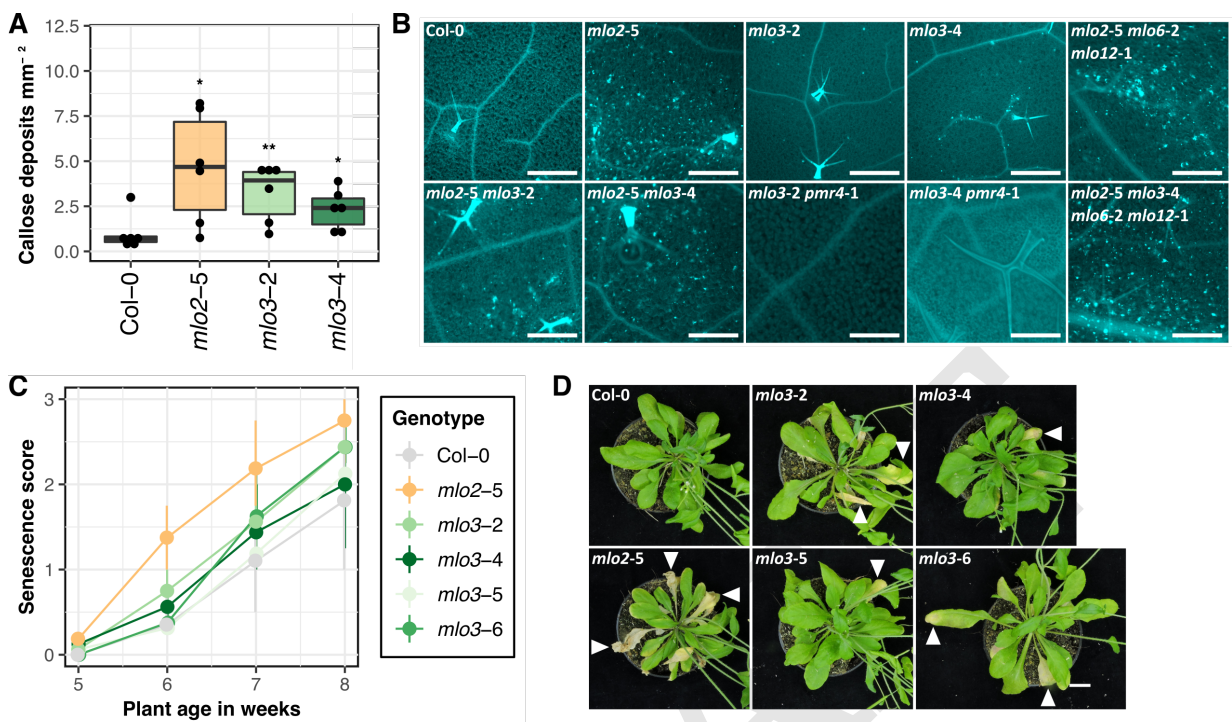

Fig. 6. Arabidopsis mlo3 exhibits spontaneous callose deposition and early leaf senescence in unchallenged rosette leaves. All leaves from six-week-old rosettes were collected and callose was stained using aniline blue. Col-0 wild type served as negative control, while the mlo2-5 single and mlo2-5 mlo6-2 mlo12-1 triple mutants were included as positive controls. We tested mlo3-2, mlo3-4, the respective double mutants with mlo2-5, mlo3-2 pmr4-1, mlo3-4 pmr4-1, and the quadruple mutant mlo2-5 mlo3-4 mlo6-2 mlo12-1. For quantitative assessment, pictures were taken with high black balance and long exposure time to minimize background, and callose deposits were quantified using CellProfiler v3.1.8 (53). A. The boxplot shows the average counts for the Col-0 wild type, mlo3-2, mlo3-4, and $m / o 2-5$ from $n=6$ independent replicates. Statistics were done with GLM (Poisson distribution). B. Representative micrographs for each genotype from (A) and Table 2); Scale bar: $500 \mu$ m. C. The senescence of each plant was rated as follows: 0 , no symptoms; 1 , mild yellowing symptoms on one or several leaves; 2 , pronounced yellowing symptoms on several leaves and/or occasional necrosis; 3 , most or all rosette leaves are necrotic. Three to four plants per genotype and replicate were assessed at five, six, seven, and eight weeks after germination; the graph was produced with the average values for each time point and replicate. The error bars indicate maximum and minimum average values. D. Representative photographs of seven-week-old rosettes of Col-0, mlo2-5, and the mlo3 mutants. Arrows indicate leaves with visible symptoms of senescence. Scale bar: $1 \mathrm{~cm}$.

Arabidopsis mlo3 single mutants do not show a significantly altered development upon exposure to osmotic stress by mannose.

\section{mlo3 mutants exhibit spontaneous callose deposition} similar to $\mathbf{m l o 2}$. In addition to enhanced powdery mildew resistance, Arabidopsis mlo2 mutant plants exhibit an early leaf senescence phenotype, which is associated with spontaneous callose deposition in rosette leaves $(4,7)$. The early senescence phenotype cannot be seen in mlo6 and mlo12 single mutants (4). We analyzed six-week-old mlo3 mutant plants grown under short day conditions for the development of spontaneous callose depositions in rosette leaves. This was done in comparison to Col-0 wild type and the mlo2-5 mutant, which served as negative and positive controls, respectively, in this set of experiments. To this end, we collected all rosette leaves from each individual plant, stained them with aniline blue, photographed randomly selected leaf spots for aniline blue-derived fluorescence, and quantified the number of deposits $\mathrm{mm}^{-2}$ in the mesophyll (Figure 6A and Figure 6B). We found very few callose deposits in Col-0 (less than $1 \mathrm{~mm}^{-2}$ ), while the mlo2-5 mutant showed elevated numbers of these deposits $\left(2-8 \mathrm{~mm}^{-2}\right)$. The mutant lines mlo3-2 and mlo3-4 displayed slightly lower numbers $\left(2-5 \mathrm{~mm}^{-2}\right)$, which were nonetheless significantly increased compared to the wild type in five out of six independent experiments. In a separate experiment, comprising also double and higher order mutants, the average callose deposits $\mathrm{mm}^{-2}$ were somewhat higher for all tested genotypes, with $\sim 6$ (Col-0), $\sim 12$ (mlo3-2) and $\sim 26$ (mlo3-4). The mlo2 mlo3 double mutants had on average $\sim 33$ and $\sim 70$ deposits $\mathrm{mm}^{-2}$, the mlo 2 mlo6 mlo12 showed $\sim 99$ deposits $\mathrm{mm}^{-2}$, and the quadruple mutant mlo2 mlo3 mlo6 mlo12 displayed $\sim 55$ deposits $\mathrm{mm}^{-2}$ in rosette leaves of six-week-old plants (Table 2, Figure 6B and Figure S6). We further crossed mlo3-2 and mlo3-4 with pmr4-1, which is deficient in a callose synthase (GLUCAN- 

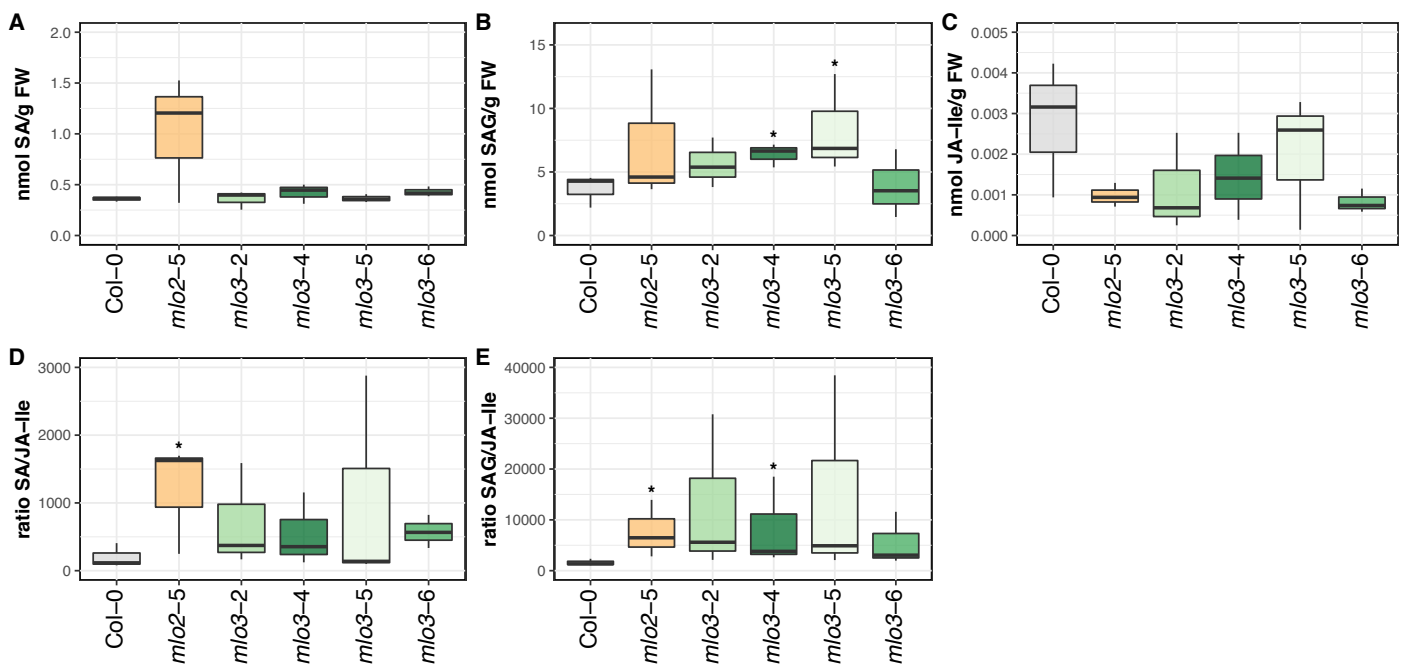

Fig. 7. Homeostasis of the phytohormone conjugates SAG and JA-Ile are mildly affected in five-week-old $m$ mlo3 mutant plants. The levels of the phytohormones (A) SA, (B) SA glucoside (SAG), and (C) JA-isoleucine (JA-lle) in the leaves of unchallenged Col-0, mlo2-5, mlo3-2, mlo3-4, mlo3-5, and mlo3-6 plants grown under a short day cycle were measured at four, five, six, and seven weeks post germination by UPLC-nano ESI-MS/MS. Only values for five-week-old plants are shown. Based on these values, the ratio of SA over JA-lle (D) and SAG over JA-lle (E) were calculated for each plant. The boxplots are based on $n=3$ independent replicates; statistical analysis was performed using GLM (quasi-Poisson distribution) and Kruskal rank sum tests.

SYNTHASE-LIKE5/POWDERY MILDEW RESISTANT4, AtGSL5/AtPMR4) that is needed for the deposition of wound and papillary callose $(54,55)$. AtPMR4 is further required for the spontaneous callose deposition phenotype of mlo2 mutant plants (4). Resulting mlo3 pmr4 double mutants showed wild type-like callose deposition in leaves of six-week-old plants, with $c a .2$ and 4 deposits $\mathrm{mm}^{-2}$, respectively (Table 2 and Figure S6).

Next, we assessed the mlo3 mutants in comparison to the wild type Col-0 and the mlo2-5 mutant for macroscopic signs of early leaf senescence under stress-free long day conditions. Col-0 did not show any visible indication of senescence up to seven weeks after germination, while the mlo2-5 mutant displayed extensive senescence symptoms (leaf chlorosis and necrosis) from six weeks onwards (Figure 6C and Figure 6D). We found pronounced senescence symptoms in all four $\mathrm{mlo3}$ mutants at seven weeks of age, although apparently less drastic than in case of the mlo2 genotype. Altogether, the data shows that Arabidopsis mlo3 mutants, like mlo2 genotypes, exhibit spontaneous and AtPMR4-dependent callose deposition and early signs of senescence in unchallenged leaves of mature rosettes.

Levels of $S A(G)$ over JA-lle are transiently increased in the leaves of mlo3 mutant plants. Enhanced SA and SA glucoside (SAG) levels were previously observed in $\mathrm{mlo} 2$ mutant plants $(11,56)$, and SA homeostasis processes were among the highly represented GO terms in the co-expression network of AtMLO3 (Figure 1C). We therefore measured levels of the phytohormones SA, jasmonic acid-isoleucine (JAIle) and abscisic acid (ABA) in rosette leaves at four, five, six, and seven weeks after sowing. We found that SA levels were significantly higher in five-week-old $\mathrm{mlo} 2$ plants compared to Col-0 (1.2 vs. $0.4 \mathrm{nmol} \mathrm{SA} / \mathrm{g} \mathrm{FW})$, while the $m l o 3$ mutant lines were mostly comparable to Col-0 (Figure 7, Figure S7, Figure S8 and Table S2). There were only a few de- tectable differences found in mlo mutant plants compared to wild type with regard to most of the jasmonates, with the exception of the JA conjugate 12-OH-JA-Ile, which is a JA-Ile degradation product and was significantly increased in a transient manner in seven-week-old mlo3-4 (4-fold), mlo3-5 (12fold), and mlo3-6 (3-fold) plants. Notably, the SA conjugate SAG was in tendency increased in five-week-old mlo3 mutant plants (mlo3-4 and mlo3-5, statistically significant with $\mathrm{p}<0.05$ ) (Figure 7, Figure S7, Figure S8 and Table S2). We had a closer look at the SA to JA-Ile and SAG to JA-Ile ratios to assess the homeostasis of these two antagonistically acting hormones. The SAG/JA-Ile ratio was significantly increased in favor of SAG in five-week-old mlo2-5 (5- and 6-fold, respectively), and between 3.5 and 9-fold in mlo3-2, mlo3-4, mlo3-5 and mlo3-6 mutant plants, compared to Col-0. At six and seven weeks, only mlo2-5 plants displayed clearly and persistently increased SAG/JA-Ile ratios (15-fold and 10fold, respectively). Taken together, mlo3 mutant plants show noticeable transient differences to wild type plants in the ratio of SAG/JA-Ile in five-week-old plants.

\section{Conclusions}

The functional role of most MLO family members remains enigmatic to date (20). Here we conducted a detailed characterization of the AtMLO3 expression pattern and tested a potential contribution of the gene to plant immunity or premature senescence. Based on comprehensive in silico analysis (public microarray data) we found that despite some overlap AtMLO3 is in part expressed in different tissues and under different conditions than AtMLO2 (Figure 1A). Experimental results obtained with a PAtMLO3::GUS reporter line were, however, largely incongruent with the public microarray data, especially regarding the elevated expression levels in senescent leaves (Figure S2). This discrepancy could be due to the fact that essential cis-regulatory sequences may reside e.g. in AtMLO3 up- or downstream regions 
and/or intronic sequences not covered by the transgenic PAtMLO3::GUS construct. It is known that in particular promoter-proximal introns in Arabidopsis genes are enriched in sequence motifs that enhance gene expression (57). These could also be critical for the native expression pattern of AtMLO3, in particular the senescence-associated expression, which was essentially lacking in the PAtMLO3::GUS transgenic line. The generally weak GUS staining obtained with the PAtMLO3::GUS transgenic line could also simply reflect the natural ( $c a$. ten-fold) differences in AtMLO2 and AtMLO3 expression levels (Table S1).

Co-expression networks can be exploited to predict gene functions (58). AtMLO2 and AtMLO3 share a considerable number of co-expressed genes (79 out of the top 300; Figure 1B). More than $30 \%$ of these co-expressed genes are functionally related to plant defense and/or SA signaling (Figure 1C). A set of genes co-expressed with AtMLO2 was previously found to represent an evolutionarily conserved regulon with a function in antifungal plant innate immunity (59). Most of the co-expressed genes share a common cis-regulatory element in their promoters, and several mutants of these genes exhibit altered pathogen infection phenotypes and perturbed levels of antifungal defense compounds (glucosinolates and glucosinolate metabolites). Since key components of this regulon with well-defined functions in exocytosis (e.g. AtSYP121/AtPEN1, AtSNAP33 and AtVAMP722) and glucosinolate biosynthesis/secretion (e.g. AtPEN2, AtPDR8/AtPEN3, AtCYP83B1) are lacking in the list of genes jointly co-expressed with AtMLO2 and AtMLO3, the here reported 79 genes may define a separate regulon with a distinctive function. Given the overrepresentation of SA-related GO terms in the 79 genes (Figure 1C), this function could be related to SA-dependent defense and/or other SA-dependent processes. Many of the putative cis-regulatory elements we identified in the upstream sequences of these genes may be binding sites for WRKY transcriptional regulators. For instance, we found potential AtWRKY18 and AtWRKY38 binding sites to be abundant (Supplementary File 3). Notably, wrkyl8 wrky 40 double mutants were shown to exhibit increased resistance to $G$. orontii accompanied by de-regulated levels of SA and JA (60), while AtWRKY38 and AtWRKY62 both function as negative regulators of SA-dependent defense against $P$. syringae (61). These findings further support a potential role for AtMLO3 in SA-dependent defense-related processes. Together with the strong transcriptional upregulation of AtMLO3 in systemic tissues following local bacterial challenge and the AtFMO1-dependent induction of AtMLO3 expression upon treatment with the SAR-like and defense priming inducer pipecolic acid (Figure S1), this may point to a putative role for AtMLO3 in SAR.

Based on the close phylogenetic relationship of AtMLO3 and the characteristics of its expression profile outlined above, we hypothesized that mlo3 mutants might show altered pathogen infection phenotypes. We selected four independent $\mathrm{mlo} 3$ T-DNA insertion mutants that each represent a null mutant (full-length transcript lacking; Figure 2) and subjected these mutants to inoculation with various phytopathogens from three kingdoms of life. These assays revealed essentially unaltered infection phenotypes upon challenge with either adapted or non-adapted powdery mildew fungi (Figure 3), the oomycete $H$. arabidopsidis (Figure 5A) and the bacterial pathogen $P$. syringae (Figure 5B and C). Notably, in contrast to mlo2 (11), even the SA-dependent SAR response to P. syringae was unaffected in mlo3 mutants (Figure 5D). Absence of a detectable pathogen infection phenotype in our set of mlo3 mutants might be explained in different ways. First, we only used biotrophic (powdery mildews, H. arabidopsidis) or hemibiotrophic (P. syringae) pathogens in our bioassays. It would be interesting to expand these experiments also to necrotrophic pathogens (e.g. Botrytis cinerea) in the future. Second, we only scored particular parameters (e.g. pathogen entry and sporulation) of each plant-pathogen interaction but did not assess others (e.g. the rate of hyphal expansion) and thus might have missed a respective (subtle) phenotype. Third, mlo3 mutants could be affected for a given parameter in a particular plant-pathogen interaction, but the effect might be too small to be recognized with the limited number of experimental replicates we performed. Finally, it of course also remains a possibility that mlo3 mutants do not show any altered infection phenotypes at all.

Apart from not presenting a distinctive alteration on their own, mlo3 mutants failed to exert a synergistic (enhancing resistance) or epistatic (relieving resistance) effect on the infection phenotype of partially ( $m l o 2$ and $m l o 2 ~ m l o 6)$ or fully (mlo2 mlo6 mlo12) powdery mildew-resistant mutant plants in any of the pathogen assays (Figure 3 and Figure S3). Unlike AtMLO6 and AtMLO12 (4) and despite the set of co-expressed genes shared with AtMLO2 (see above), AtMLO3 therefore does not seem to exhibit any cryptic and/or unequal genetic redundancy with AtMLO2 regarding plant immunity. The absence of any pathogen phenotype in mlo3 mutants might be considered rather unexpected given the plant immunity-related expression profile and co-expression network of the AtMLO3 gene. However, it is consistent with the fact that so far all mlo mutants associated with altered pathogen defense have been ascribed to members of the phylogenetic clades IV (monocotyledonous plants) and V (dicotyledonous plants) of the MLO family, while $A t \mathrm{MLO} 3$ is a member of clade VI $(3,19)$.

In unchallenged leaf epidermal cells, the barley Mlo protein localizes evenly to the cell periphery (plasma membrane) but accumulates in discrete areas upon attempted attack by the powdery mildew pathogen (62). Such pathogen-triggered redistribution and focal accumulation at fungal attack sites has been seen in the case of several but not all tested plasma membrane-resident proteins (62). For Arabidopsis AtPEN1 and AtPEN3 it was shown that both actin-dependent and -independent processes contribute to the recruitment of these proteins to regions of biotic stress (63). The focal accumulation of proteins at these sites correlates with their extracellular incorporation into local pathogen-induced cell wall compartments (papillae; (64)). While the AtMLO2 pro- 
tein, reminiscent of barley Mlo, shows focal accumulation at fungal pathogen attack sites, the AtMLO3 protein lacks this dynamic behavior and remains evenly distributed at the cell periphery following powdery mildew challenge (Figure 4). This result suggests that the capacity for pathogen-triggered focal accumulation is not an intrinsic feature of all MLO proteins but an isoform-specific attribute. Specific peptide motifs in the protein, certain post-translational modifications and/or association with particular interaction partners may determine the membrane dynamics of individual MLO paralogs.

Powdery mildew-resistant barley mlo mutants are well known for their premature senescence phenotype, which is associated with spontaneous callose deposition and catabolism of photosynthetic pigments $(5,6,8)$. Arabidopsis mlo2 mutants $(4,7)$ and wheat mlo null mutants $(65)$ share this undesired feature. Results of genetic analyses revealed that at least in Arabidopsis this phenotype is fully dependent on SA biosynthesis and signaling, as mlo2 double mutants with sid2, pad4 or nprl result in restored wild type-like leaf senescence (4). Given the SA-related co-expression profile of AtMLO3 (Figure 1C) we explored whether mlo3 T-DNA mutants show likewise any signs of early leaf senescence. We found that mlo3 mutants, reminiscent of mlo2 mutants, exhibit both premature leaf chlorosis/necrosis (Figure 6C-D) and spontaneous callose deposition in the absence of any pathogen (Figure 6A-B). The extent of the latter phenotype is, however, less pronounced than in the mlo2-5 null mutant and thus quantitatively intermediate between the Col-0 wild type and the mlo2-5 genotype (Figure 6A). Similar to powdery mildew infection (Figure 3), the absence of AtMLO3 did not result in any detectable synergistic or epistatic effect regarding spontaneous callose deposition of the mlo2 mutant (Figure 6B and Table 2). However, putative synergism in mlo2 mlo3 double mutants could be masked by the comparatively small contribution of the mlo3 mutation to the overall phenotype. By contrast, we noted that additional mutations in AtMLO6 and AtMLO12 appear to raise the number of callose deposits in the background of the mlo2-5 mutant, pointing to genetic redundancy of AtMLO2, AtMLO6 and AtMLO12 regarding this phenotype. In case of mlo2 mutants, the callose synthase AtGSL5/AtPMR4 is required for the formation of callose deposits - mlo2 pmr4 double mutants lack this feature (7). We demonstrate by $m l o 3 ~ p m r 4$ double mutant analysis that analogously AtGSL5/AtPMR4 is also required for spontaneous callose deposition in $\mathrm{mlo3}$ mutant plants (Figure 6B and Table 2). This suggests a common signaling pathway leading to aberrant callose deposits in the absence of AtMLO2 or AtMLO3. Our findings further support the previous notion that spontaneous callose deposition is not causally linked to the powdery mildew resistance trait. In the case of the mlo2 mutant, both aspects can be genetically uncoupled, as for example demonstrated by $m l o 2$ sid 2 , mlo 2 pmr 4 and $m l o 2 ~ r c d l$ double mutants, which all exhibit resistance but lack the callose phenotype $(4,12)$. Conversely, mlo3 mutants shows spontaneous callose deposition but lack enhanced powdery mildew resistance.
In Arabidopsis, a developmentally controlled increase in SA and SAG levels in the rosette leaves of $m l o 2$ and $m l o 2$ mlo6 mlo12 mutant plants precede the premature senescence phenotype $(4,56)$. As previously demonstrated by genetic analysis, SA/SAG accumulation is required for the occurrence of both, signs of leaf senescence and spontaneous callose deposition in mlo2 mutants (4). We thus determined the levels of various phytohormones, including SA/SAG and the antagonistically acting JA and some of its derivatives, in the course of plant development. Results of this experiment confirmed the previously reported (56) developmentally controlled increase in SA/SAG levels in the mlo2 mutant in comparison to the Col-0 wild type (Figure 7A and B). We also found transiently (in five-week-old plants) increased SAG levels and concomitantly reduced JA-Ile levels in mlo3 mutant plants, resulting in a markedly increased SAG/JA-Ile ratio compared to Col-0 wild type (Figure 7E). Unlike the mlo2-5 mutant, which apart from an increased SAG/JA-Ile also showed an enhanced SA/JA-Ile ratio, the SA to JA-Ile relation was essentially wild type-like in the mlo3 mutants (Figure 7D). It thus seems as if the mlo3 mutants represent a weakened form of the mlo2 mutant regarding alterations in leaf phytohormone content, with the conjugated forms (SAG and JA-Ile), but not with the unconjugated SA showing aberrant accumulation during plant development. Notably, in contrast to the mlo2 mutant, this effect is transient in the mlo3 mutant plants, which likely explains the much less pronounced callose phenotype seen in these genotypes (Figure 6A-B).

In summary, our study revealed dissimilar roles for AtMLO2 and $A t M L O 3$ in pathogen defense but functional overlap between the two genes regarding the control of timely leaf senescence. Both genes/proteins might act cooperatively in a shared pathway involving SA signaling. Absence of either gene results in spontaneous AtGSL5/AtPMR4-dependent callose deposition and premature leaf chlorosis and necrosis. It thus seems as if the clade VI protein $A t \mathrm{MLO} 3$ in part functionally diverged from the clade $\mathrm{V}$ protein $A t \mathrm{MLO} 2$ after phylogenetic separation, yet both retained a shared role in development. It remains, however, a formal possibility that mlo3 mutants are affected in age-related resistance (ARR). ARR describes the phenomenon of enhanced immunity in mature plants as compared to young plants (66). It is effective against pathogens from different kingdoms of life and was shown to depend on SA accumulation $(67,68)$. Given their premature senescence, the co-expression of genes associated with SA-related processes and the altered phytohormone balance, mlo3 and mlo2 mutants might show aberrant ARR - a possibility that needs to be tested experimentally in the future. Since preliminary evidence indicates that MLO proteins may have the capacity to form hetero-oligomeric complexes (69), it also remains to be seen whether AtMLO2 and AtMLO3 operate separately in distinct protein assemblies or at least in part act cooperatively in a common complex. 


\section{Material and Methods}

In silico expression analysis. Arabidopsis gene expression data for AtMLO1 (At4g02600), AtMLO2 (At1g11310), AtMLO3 (At3g45290), AtMLO6 (At1g61560), and AtMLO12 (At2g39200) was extracted from the eFP browser (http://bar.utoronto.ca/efp/cgi-bin/efpWeb.cgi; (27)) in January 2019. Co-expression network analysis was done using ATTED-II release 2017.12.14 (http://atted.jp; (28)). GO terms were extracted with PANTHER v10, release 2018_04 (30) and PLAZA v4.0 (29) was used for GO term enrichment analysis, using false discovery rate (FDR) at $\mathrm{p}<0.05$ and Fisher's exact test. We used AthaMap $(43,44)$ and MEME suite v5.0.4 (45) for the detection of putative cis-regulatory elements.

Plant materials. We used four independent T-DNA insertion lines for AtMLO3 in the background of the accession Col-0, i.e. mlo3-2 (SALK_063243; (4)), mlo3-4 (SALK_027770), mlo3-5 (GK_787G01), and mlo3-6 (SALK_116848). The novel lines were acquired from the Nottingham Arabidopsis Seed Centre (NASC) and screened for presence of T-DNA according to (70). We further included the wild type accessions Columbia (Col-0) and Landsberg erecta (L-er), and the following mutants (all in Col-0 background): mlol-6, mlo2-5 and mlo2-5 mlo6-2 mlo12-1 (4), pen2-1 pad4-1 sag101-1 (46), eds 1-2 (48). Combinations of mlo3-2 and mlo3-4 with mlo2-5, mlo6-2, or mlo12-1 were generated by crossing of the respective T-DNA insertion lines. Both mlo3-2 and mlo3-4 were also combined with pmr4-1 (55). The previously described transgenic promoter-GUS lines PAtMLO2::GUS and PAtMLO3::GUS (22) were used for the GUS assays.

Plant growth conditions. Arabidopsis plants were grown on SoMi513 soil (HAWITA, Vechta, Germany) in 9x9 cm pots or round pots $(\mathrm{r}=3 \mathrm{~cm})$ under a short day cycle $(10 \mathrm{~h}$ light period set to $22{ }^{\circ} \mathrm{C}, 14 \mathrm{~h}$ darkness at $20^{\circ} \mathrm{C}$, at 80 $90 \%$ relative humidity $(\mathrm{RH})$ and a light intensity of $80-100$ $\mu \mathrm{mol} \mathrm{s} \mathrm{m}^{-2}$ ) until transferring them to the respective plant pathogen growth chamber. Conditions in the H. arabidopsidis chamber were $16^{\circ} \mathrm{C}, 6065 \% \mathrm{RH}$ and a light intensity of $100 \mu \mathrm{mol} \mathrm{s} \mathrm{m}^{-1} \mathrm{~m}^{-2}$ set to a short day cycle $(8 \mathrm{~h}$ light, $16 \mathrm{~h}$ dark period). G. orontii-infected plants were grown under a short day cycle at $10 \mathrm{~h}$ light period and $14 \mathrm{~h}$ darkness at $20^{\circ} \mathrm{C}, \mathrm{ca}$. $65 \% \mathrm{RH}$ and a light intensity of $100 \mu \mathrm{mol} \mathrm{s} \mathrm{s}^{-1}$ $\mathrm{m}^{-2}$. For $E$. pisi infection assays, conditions were a long day cycle $12 \mathrm{~h}$ light and $12 \mathrm{~h}$ dark period at $21^{\circ} \mathrm{C}$ with the light intensity at $100 \mu \mathrm{mol} \mathrm{s}^{-1} \mathrm{~m}^{-2}$ and ca. $60 \% \mathrm{RH}$. For SAR assays, plants were grown in individual pots $(5 \times 5 \times 6 \mathrm{~cm})$ filled with a mixture of soil (Einheitserde CL ED73), vermiculite, and sand (8:1:1) and cultivated in a separate chamber in a short day cycle $\left(10 \mathrm{~h} \mathrm{light} \mathrm{at} 80-100 \mu \mathrm{mol} \mathrm{s}^{-1} \mathrm{~m}^{-2} / 14 \mathrm{~h}\right.$ dark) at $20{ }^{\circ} \mathrm{C}$ and $60-68 \%$ RH. For flowering/seed production and for GUS staining assays (see below), Arabidopsis plants were transferred to a long day chamber six weeks after germination; long day conditions were 16 light at $23^{\circ} \mathrm{C}, 8 \mathrm{~h}$ darkness at $20{ }^{\circ} \mathrm{C}$, with $60-65 \% \mathrm{RH}$ and the light intensity was at $105-120 \mu \mathrm{mol} \mathrm{s}{ }^{-1} \mathrm{~m}^{-2}$. Plants for senescence assays were exclusively grown under long day conditions.

Gene expression analysis by RT-PCR. Leaves were collected from five-week-old Arabidopsis plants grown under a short day cycle, flash-frozen in liquid $\mathrm{N}_{2}$ and milled to fine powder at $30 \mathrm{~s}^{-1}$ for $30 \mathrm{~s}$. RNA isolation was performed using the NucleoSpin ${ }^{\circledR}$ RNA Plant kit (Macherey-Nagel, Düren, Germany) following the manufacturer's instructions, and RNA integrity was determined by agarose gel electrophoresis. cDNA synthesis was done with 500 ng RNA using the High-Capacity RNAto-cDNA kit (Applied Biosystems/Thermo Scientific). The RT-PCR was performed with $T a q$ polymerase at an annealing temperature of $54{ }^{\circ} \mathrm{C}$, an elongation time of $60 \mathrm{~s}$ (for AtMLO3) or $90 \mathrm{~s}$ (for AtMLO2), and 35 cycles. Primers for AtMLO3 RT-PCR were AtMLO_3C (forward primer), 5'-GAAAATGGAATTCGTGGGAGAAAGAG3' and AtMLO_331 (reverse primer), 5'GCTAATACAAGTCGTGTGATTATG-3', resulting in a 697 bp product. Primers for AtMLO2 RT-PCR were AtMLO_2C, 5'-ATGGAGATGGAGATAAACCCGGTC3' (forward primer) and AtMLO_2bw1, 5'ACTAGTATCTAGGAGAAGGAG-3' (reverse primer), generating a product of $1,209 \mathrm{bp}$.

GUS staining. The plant tissue was submerged in GUS staining solution $\left(39 \mathrm{mM} \mathrm{NaH} \mathrm{PO}_{4}, 61 \mathrm{mM} \mathrm{Na} 2 \mathrm{HPO}_{4}\right.$, $2 \mathrm{mM} \mathrm{K}_{3} \mathrm{Fe}(\mathrm{CN})_{6}, 0.5 \mathrm{mg} \mathrm{mL}^{-1} \mathrm{X}$-Gluc (Apollo Scientific, Whitefield, UK), $0.1 \% \mathrm{v} / \mathrm{v}$ Triton X-100) and vacuum was applied three times for $10 \mathrm{~min}$. Then, seedlings were incubated in the solution for 2-3 h, while plant rosettes were incubated overnight (up to $16 \mathrm{~h}$ ), both at $37{ }^{\circ} \mathrm{C}$. Subsequently, the samples were bleached in $80 \%$ ethanol at room temperature for at least two to three days. Photographs of GUSstained seedlings or of GUS-stained leaves after pathogen infection were taken using the Keyence Biorevo BZ 9000 microscope (Keyence Corporation, Osaka, Japan), rosettes were photographed with a Coolpix P600 camera (Nikon, Amsterdam, The Netherlands).

Callose staining. Arabidopsis plants were grown under a short day cycle for six weeks. Then, all leaves from the rosette were collected in $80 \%$ ethanol for bleaching of the chlorophyll for at least three days at room temperature. Thereafter, the leaves were submerged in aniline blue staining solution $\left(150 \mathrm{mM} \mathrm{K} \mathrm{HPO}_{4}, 0.01 \%\right.$ w/v aniline blue; Sigma-Aldrich, Munich, Germany) in the dark overnight. For microscopic assessment, the leaves were first mounted on a glass slide. The samples were analyzed by fluorescence microscopy with a Keyence Biorevo BZ 9000 microscope (Keyence Corporation), using UV excitation (327-427 nm) and the DAPI/Aniline blue emission filter (emission 417$477 \mathrm{~nm}$ ). Pictures were taken with the BZII-viewer software, set to long exposure time (1/7 to $1 / 3 \mathrm{~s}$ ) and high black balance to remove background fluorescence. Callose deposit counts were performed using CellProfiler v3.1.8 software for batch analysis (53). 
Powdery mildew infection assays. Arabidopsis plants were inoculated with powdery mildew at $c a . r=2.5 \mathrm{~cm}$ in rosette size at four to five weeks after germination. Golovinomyces orontii is adapted to infection of Arabidopsis and was cultivated on susceptible eds 1-2 plants; Erysiphe pisi was cultivated on susceptible pea isolates. Inoculation was conducted by leaf-to-leaf transfer of conidiospores. Leaves from at least four individual plants were collected for each replicate at $48 \mathrm{hpi}$, and five independent replicates were performed for each assay. The leaves were bleached in $80 \%$ ethanol at room temperature for at least two to three days. Prior to microscopic analysis, fungal structures were stained by submerging the leaves in Coomassie staining solution $(45 \% \mathrm{v} / \mathrm{v}$ methanol, $10 \% \mathrm{v} / \mathrm{v}$ acetic acid, $0.05 \%$ w/v Coomassie blue R-250; Carl Roth, Karlsruhe, Germany) twice for 15-30 s and shortly washed in tap water in between and thereafter. The samples were analyzed with an Axiophot microscope (Carl Zeiss AG, Jena, Germany). The fungal penetration rate was determined as the percentage of spores successfully developing secondary hyphae over all spores that attempted penetration, visible by an appressorium. Macroscopic pictures of $G$. orontii-infected plants were taken at 7 dpi with a Coolpix P600 camera (Nikon).

\section{Hyaloperonospora arabidopsidis infection assays.} Arabidopsis seedlings were grown on a $c a$. 10:1 soil-sand mix sterilized by microwaving for $15 \mathrm{~min}$. The seedlings were grown in a thin lawn, and three pots per genotype $(\mathrm{r}=3 \mathrm{~cm})$ were used for each replicate. The seeds were stratified at $4{ }^{\circ} \mathrm{C}$ in the dark for two days and then grown under a long day cycle, covered by a lid for eight days. The plants were inoculated with $\mathrm{Hpa}$ Noco2 by spraying a spore suspension $\left(4 \times 10^{4}\right.$ spores $\left.\mathrm{mL}^{-1}\right)$ with four to five spray boosts onto each pot twice with a pause of 20-30 $\mathrm{min}$ in between. Then, the plants were incubated under a short day cycle for seven days. Thereafter, all plants from each pot were collected to determine the fresh weight with a fine balance. Subsequently, the spores were washed off with 1-2 $\mathrm{mL}$ of distilled water. The spore concentration was calculated using a hemocytometer by averaging the counts from ten of the $1 \mathrm{~mm}^{2}$ squares. The infection success was expressed as spores per gram fresh weight, determined according to the following formula:

$$
\frac{\text { mean }_{\text {counts }} * V_{\mathrm{H}_{2} \mathrm{O}}[\mathrm{mL}] * 10^{4}}{\text { freshweight }[\mathrm{g}]}
$$

Pseudomonas syringae infection assays. Arabidopsis seedlings were grown as a thin lawn, as described above for Hpa infection assays. The seedlings were cultivated under a short day cycle for 15 days and then placed in the dark for one day before spray-inoculation of bacteria. Prior to inoculation, the Pst strain DC3000 bacteria were suspended in $150 \mu \mathrm{L}$ of $10 \mathrm{mM} \mathrm{MgCl}_{2}$, plated on NYG-Rifampicin plates, and then grown at $28{ }^{\circ} \mathrm{C}$ overnight. Afterwards, the bacteria were collected from the plates and re-suspended in $2 \mathrm{~mL}$ of $10 \mathrm{mM}$ $\mathrm{MgCl}_{2}$ and diluted to an optical density at $600 \mathrm{~nm}\left(\mathrm{OD}_{600}\right)$ of 0.2. Directly before spray inoculation, Silwet L-77 was added to a concentration of $0.04 \% \mathrm{v} / \mathrm{v}$. Each pot was sprayed ten times with the bacteria suspension. Inoculated plants were placed in the dark for another 3-4 h before collecting 0 dpi samples. Afterwards, the plants were cultivated in a short day cycle. The bacterial titer was determined at $0 \mathrm{dpi}$ as inoculation control, and at $3 \mathrm{dpi}$ as follows. All plants from one pot of each genotype were collected, weighted, surfacesterilized by dipping them in $70 \%$ ethanol, and sampled into $1.5 \mathrm{~mL}$ of $10 \mathrm{mM} \mathrm{MgCl} 2$ containing $0.01 \%$ Silwet L-77. The bacteria were extracted by shaking at $1,500 \mathrm{rpm}$ on a platform vortex for $1 \mathrm{~h}$. Then, a dilution series of $10^{0}$ to $10^{-5}$ was prepared. From each dilution, $5 \mu \mathrm{L}$ (accounting for a dilution factor of 150) were drop-inoculated on a NYG-Rifampicin plate and the plates were incubated at $28{ }^{\circ} \mathrm{C}$ for $c a$. one and a half days. The bacterial titer was expressed as $\log 10$ of colony-forming units (CFU) per gram fresh weight using the following formula:

$$
\log _{10}\left(\frac{\text { dilution factor } * \text { colony count } * 10^{X}}{\text { fresh weight }[\mathrm{g}]}\right)
$$

Systemic acquired resistance assays with Pseudomonas syringae pv. maculicola lux. To monitor their SAR response, Arabidopsis mlo3-4, mlo3-6, mlo2-5, and Col- 0 wild type plants were grown in controlled short day conditions $\left(10 \mathrm{~h} \mathrm{light} / 14 \mathrm{~h}\right.$ dark at $\left.20{ }^{\circ} \mathrm{C}\right)$. The soil in which the plants were grown was microwaved for 15 min. P. syringae pv. maculicola strain ES4326 (Psm) and Psm constitutively expressing the Photorhabdus luminescens luxCDABE operon (Psm lux; (52)) were grown at $28{ }^{\circ} \mathrm{C}$ on King's B plates containing the appropriate antibiotics. For the respective leaf inoculations, liquid King's B cultures including antibiotics were inoculated with bacteria and grown overnight at $28{ }^{\circ} \mathrm{C}$. After collecting the bacteria, they were washed in $10 \mathrm{mM} \mathrm{MgCl}$ three times and finally diluted to the respective optical density mentioned below. SAR experiments were conducted on five-week-old naïve plants of healthy appearance. At day one, three lower mature leaves were infiltrated from the abaxial site with either a Psm dilution of $\mathrm{OD}_{600}$ at 0.005 or $10 \mathrm{mM} \mathrm{MgCl} 2$ (mock control) using a needleless $1 \mathrm{~mL}$ syringe. Two days later, three upper mature (i.e., systemic) leaves of the pretreated plants were inoculated with a Psm lux dilution of $\mathrm{OD}_{600}$ at 0.0005 in the same way. Growth of Psm lux in the challenged leaves was monitored three days later using a luminometer as described in (9). Three leaves per plant were measured and after subtracting the average luminescent background, they were averaged to a value per plant expressed in relative light units (RLU) per $\mathrm{cm}^{2}$. The bioluminescence of Psm lux measured in RLUs is linearly correlated with the growth expressed in CFU (11, 52). At least seven plants per genotype and treatment were analyzed and the experiment was conducted twice.

Transient overexpression via particle delivery system. Four-week-old Arabidopsis plants were transformed using the PDS-1000/He HeptaTM device (Bio-Rad, Munich, Germany) equipped with a $900-1100$ psi (i.e., 6.2 to $7.6 \mathrm{MPa}$ ) rupture disc. Five to seven plants grown under a short day cycle covered by a lid were removed from the soil, the roots 
were washed with sterile water and then the plants were placed on $1 \%$ agar containing benzimidazole. Plasmid DNA purified from a $200 \mathrm{~mL}$ culture using the NucleoBond ${ }^{\circledR}$ Xtra MIDI kit (Macherey-Nagel) following the manufacturer's instructions and diluted to $1 \mu \mathrm{g} \mu \mathrm{L}^{-1}$ was used for coating of the gold particles. Then, $5 \mu \mathrm{g}$ of plasmid DNA were bound to $3 \mathrm{mg}$ gold micro-carriers ( $1 \mu \mathrm{m}$, Bio-Rad) by adding $50 \mu \mathrm{L}$ of $2.5 \mathrm{M} \mathrm{CaCl}_{2}$ and $20 \mu \mathrm{L}$ of $0.1 \mathrm{M}$ spermidine, followed by vortexing on a platform vortex at 1,500 rpm for $3 \mathrm{~min}$. The gold was pelleted by centrifugation for $2 \mathrm{~s}$, first washed with $140 \mu \mathrm{L}$ of mbox $70 \%$ ethanol and then with $140 \mu \mathrm{L}$ of $100 \%$ ethanol. The carriers were suspended in $60 \mu \mathrm{L}$ of $100 \%$ ethanol. Each of the seven macro-carriers was loaded with $7 \mu \mathrm{L}$ of the carrier suspension. Particle delivery was carried out as recommended by the PDS-1000/He manual, applying vacuum at -24 inch $\mathrm{Hg}(-81 \mathrm{kPa})$ for Arabidopsis with the plate in the lowest level of the device. Plants were subsequently placed in the G. orontii infection chamber for recovery and inoculated with $G$. orontii spores one day later. Leaf samples were analyzed by confocal laser scanning microscopy at $2 \mathrm{dpi}$.

Confocal laser scanning microscopy. Confocal laser scanning microscopy was conducted with a Leica TCS SP8 using the LAS-X software package (Leica microsystems, Wetzlar, Germany). Arabidopsis leaves were fixed on glass slides by use of double-sided tape. Fluorescence pictures and Z-stacks were recorded with the 20x or $63 \mathrm{x}$ water immersion objective (Leica). Fluorescence was recorded with the following specifications: YFP excitation at $514 \mathrm{~nm}$ (argon laser), emission at 525-570 nm.

Osmotic stress test. Arabidopsis seeds were sterilized as follows: around 50-100 seeds were placed in a reaction tube column and submerged in $500 \mu \mathrm{L}$ of $70 \%$ ethanol for $1-2 \mathrm{~min}$ at room temperature. Next, the column was centrifuged at $1,500 \mathrm{~g}$ for $1 \mathrm{~min}$ and the flow-through was discarded. The seeds were then washed with $500 \mu \mathrm{L}$ of $100 \%$ ethanol, centrifuged again for $1 \mathrm{~min}$ and after removal of the flowthrough, dried by another centrifugation for $2 \mathrm{~min}$. Seeds sterilized by this procedure were sown on $1 / 2$ MS agar plates without (control) or containing $300 \mathrm{mM}$ mannitol to apply osmotic stress. Seeds were vernalized at $4{ }^{\circ} \mathrm{C}$ in the dark overnight and then seedlings were grown under a short day cycle for three weeks, with plates being placed upright in a rack. Phenotyping was performed by measuring the root length, number of lateral roots, fresh weight, and by counting the number of leaves.

\section{Phytohormone determination by nanoflow Ultrahigh- -Pressure Liquid Chromatography-Tandem Mass Spectrometry (UPLC-nano ESI-MS/MS). Extraction} was performed as previously described for lipids, with some modifications (71). Plant material (100 mg) was extracted with $0.75 \mathrm{~mL}$ of methanol containing $10 \mathrm{ng}$ D4-SA, $10 \mathrm{ng}$ D5-JA (both from C/D/N Isotopes Inc., Pointe-Claire, Canada), 10 ng D4-JA-Leu (kindly provided by Otto Miersch, Halle/Saale, Germany), each as internal standard. After vortexing, $2.5 \mathrm{~mL}$ of methyl-tert-butyl ether (MTBE) were added and the extract was shaken for $1 \mathrm{~h}$ at room temperature. For phase separation, $0.6 \mathrm{~mL}$ water were added. The mixture was incubated at room temperature for $10 \mathrm{~min}$ and centrifuged at $450 \mathrm{~g}$ for $15 \mathrm{~min}$. The upper phase was collected and the lower phase was re-extracted with $0.7 \mathrm{~mL}$ methanol/water $(3: 2.5, \mathrm{v} / \mathrm{v})$ and $1.3 \mathrm{~mL}$ MTBE as described above. The combined upper phases were dried under streaming nitrogen and resuspended in $100 \mu \mathrm{L}$ of acetonitrile/water $(20: 80, \mathrm{v} / \mathrm{v})$ containing $0.3 \mathrm{mmol} \mathrm{L}^{-1}$ $\mathrm{NH}_{4} \mathrm{HCOO}$ (adjusted to $\mathrm{pH} 3.5$ with formic acid).

Reversed phase separation of constituents was achieved by UPLC using an ACQUITY UPLC ${ }^{\circledR}$ system (Waters Corp., Milford, MA, USA) equipped with an ACQUITY UPLC ${ }^{\circledR}$ HSS T3 column (100 mm x $1 \mathrm{~mm}, 1.8 \mu \mathrm{m}$; Waters Corp., Milford, MA, USA). Aliquots of $10 \mu \mathrm{L}$ were injected in a partial loop with needle overfill mode. Elution was adapted according to (72). Solvent A and B were water and acetonitrile/water $(90: 10, \mathrm{v} / \mathrm{v})$, respectively, both containing $0.3 \mathrm{mmol} \mathrm{L}^{-1} \mathrm{NH}_{4} \mathrm{HCOO}$ (adjusted to $\mathrm{pH} 3.5$ with formic acid). The flow rate was $0.16 \mathrm{~mL} \mathrm{~min}^{-1}$ and the separation temperature was constantly at $40{ }^{\circ} \mathrm{C}$. Elution was performed isocratically for $0.5 \mathrm{~min}$ at $10 \%$ solution $\mathrm{B}$, followed by a linear increase to $40 \%$ solution B in $1.5 \mathrm{~min}$; this condition was held for $2 \mathrm{~min}$, followed by a linear increase to $95 \%$ solution $\mathrm{B}$ in $1 \mathrm{~min}$; this condition was held for $2.5 \mathrm{~min}$. The column was re-equilibrated to start conditions in $3 \mathrm{~min}$.

Nanoelectrospray ionization (nanoESI) analysis was achieved using a chip ion source (TriVersa Nanomate ${ }^{\circledR}$; Advion BioSciences, Ithaca, NY, USA). For stable nanoESI, $70 \mu \mathrm{L} \mathrm{min}{ }^{-1}$ of 2-propanol/acetonitrile/water (70:20:10, $\mathrm{v} / \mathrm{v} / \mathrm{v}$ ) containing $0.3 \mathrm{mmol} \mathrm{L}^{-1} \mathrm{NH}_{4} \mathrm{HCOO}$ (adjusted to $\mathrm{pH} 3.5$ with formic acid) delivered by a Pharmacia 2248 HPLC pump (GE Healthcare, Munich, Germany) were added just after the column via a mixing tee valve. By using another post column splitter $502 \mathrm{~nL} \mathrm{~min}^{-1}$ of the eluent were directed to the nanoESI chip with $5 \mu \mathrm{m}$ internal diameter nozzles. Ionization voltage was set to $-1.7 \mathrm{kV}$. Phytohormones were ionized in a negative mode and determined in scheduled multiple reaction monitoring mode with an AB Sciex 4000 QTRAP ${ }^{\circledR}$ tandem mass spectrometer (AB Sciex, Framingham, MA, USA). Mass transitions were as previously described (73), with some modifications and were as listed in Table S3. The mass analyzers were adjusted to a resolution of $0.7 \mathrm{amu}$ full width at half-height. The ion source temperature was $40{ }^{\circ} \mathrm{C}$, and the curtain gas was set to 10 (given in arbitrary units). Quantification was carried out using a calibration curve of intensity $(\mathrm{m} / \mathrm{z})$ ratios of [unlabeled]/[deuterium-labeled] vs. molar amounts of unlabeled (0.3-1000 pmol).

Statistical analysis of the data. The statistics program $R$ v.3.5.1 (R foundation, www.r-project.org) was used for statistics and generation of boxplots. Plotting was done with the ggplot function from the ggplot2 library (74). Due to the nature of our data, i.e. non-normal distribution, unequal variance, and small sample sizes of usually maximally six independent replicates, non-parametric statistical testing was 
bioRxiv preprint doi: https://doi.org/10.1101/558122; this version posted February 22, 2019. The copyright holder for this preprint (which was not certified by peer review) is the author/funder. All rights reserved. No reuse allowed without permission.

devised. Statistical analyses were performed via Generalized Linear Modeling (GLM) assuming Poisson or binomial distribution as indicated accordingly (75), and supported by Wilcoxon-Mann-Whitney rank sum test if at least four independent replicates were available for testing. For phytohormone measurements, Kruskal rank sum tests were performed in addition.

\section{ACKNOWLEDGEMENTS}

This study was supported by the German Research Foundation (Deutsche Forschungsgemeinschaft; DFG) [grant PA861/11-1 to R.P. and grant INST 186/8221 to I.F.]. H. arabidopsidis Noco2 was kindly provided by Jane Parker (Max Planck institute for Plant Breeding Research, Cologne, Germany). The two P. syringae pv. maculicola strains were kindly provided by Jürgen Zeier (Heinrich Heine University, Düsseldorf, Germany). This work would not have been possible without coffee and chocolate.

\section{AUTHOR CONTRIBUTIONS}

S.K. and S.T. performed the pathogen assays, K.G. did the SAR experiments. S.K. and A.R. conducted callose, senescence, GUS and osmotic stress assays. Phytohormone measurements were performed by K.Z. and K.Z. and I.F. analyzed the data. S.K. did the expression analysis, statistical testing, and plotting of the data. R.P. and S.K. designed the project and wrote the manuscript. All authors have read and approved the manuscript.

\section{COMPETING FINANCIAL INTERESTS}

The authors declare that the research was conducted in the absence of any commercial or financial relationships that could be construed as a potential conflict of interest.

\section{Bibliography}

1. Jørgen Helms Jørgensen. Discovery, characterization and exploitation of Mlo powdery mildew resistance in barley. Euphytica, 63:141-152, 1992. ISSN 1573-5060.

2. Rainer Büschges, Karin Hollricher, Ralph Panstruga, Guus Simons, Marietta Wolter, Adrie Frijters, Raymond van Daelen, Theo van der Lee, Paul Diergaarde, John Groenendijk, Sonja Töpsch, Pieter Vos, Francesco Salamini, and Paul Schulze-Lefert. The barley Mlo gene: A novel control element of plant pathogen resistance. Cell, 88(5):695-705, 1997. ISSN 00928674. doi: 10.1016/S0092-8674(00)81912-1.

3. Stefan Kusch and Ralph Panstruga. Mlo-based resistance: An apparently universal "weapon" to defeat powdery mildew disease. Molecular Plant-Microbe Interactions, 30(3): 179-189, 2017. ISSN 0894-0282. doi: 10.1094/MPMI-12-16-0255-CR.

4. Chiara Consonni, Matthew E. Humphry, H. Andreas Hartmann, Maren Livaja, Jörg Durner, Lore Westphal, John P Vogel, Volker Lipka, Birgit Kemmerling, Paul Schulze-Lefert, Shauna C. Somerville, and Ralph Panstruga. Conserved requirement for a plant host cell protein in powdery mildew pathogenesis. Nature Genetics, 38(6):716-720, 2006. ISSN 1546-1718. doi: 10.1038/ng1806.

5. Marietta Wolter, Karin Hollricher, Francesco Salamini, and Paul Schulze-Lefert. The mlo resistance alleles to powdery mildew infection in barley trigger a developmentally controlled defence mimic phenotype. Molecular and General Genetics, 239(1):122-128, 1993. ISSN 0026-3925.

6. Christoph Peterhänsel, Andreas Freialdenhoven, Joachim Kurth, R. Kolsch, and Paul Schulze-Lefert. Interaction analyses of genes required for resistance responses to powdery mildew in barley reveal distinct pathways leading to leaf cell death. The Plant Cell, 9 (8):1397-1409, 1997. ISSN 1040-4651

7. Chiara Consonni, Paweł Bednarek, Matthew E. Humphry, Fedra Francocci, Simone Ferrari, Anne Harzen, Emiel Ver Loren van Themaat, and Ralph Panstruga. Tryptophan-derived metabolites are required for antifungal defense in the Arabidopsis mlo2 mutant. Plant Physiology, 152(3):1544-1561, 2010. ISSN 0032-0889. doi: 10.1104/pp.109.147660.

8. Pietro Piffanelli, Fasong Zhou, Catarina Casais, James Orme, Birgit Jarosch, Ulrich Schaffrath, Nicholas C. Collins, Ralph Panstruga, and Paul Schulze-Lefert. The barley MLO modulator of defense and cell death is responsive to biotic and abiotic stress stimuli. Plant Physiology, 129(3):1076-1085, 2002. ISSN 0032-0889. doi: 10.1104/pp.010954.

9. Johanna Acevedo-Garcia, Katrin Gruner, Anja Reinstädler, Ariane Kemen, Eric Kemen, Lingxue Cao, Frank L. W. Takken, Marco U. Reitz, Patrick Schäfer, Richard J. O'Connell, Stefan Kusch, Hannah Kuhn, and Ralph Panstruga. The powdery mildew-resistant Arabidopsis mlo2 mlo6 mlo12 triple mutant displays altered infection phenotypes with diverse types of phytopathogens. Scientific Reports, 7(1):9319, 2017. ISSN 2045-2322. doi: 10.1038/s41598-017-07188-7.

10. Birgit Jarosch, Karl-Heinz Kogel, and Ulrich Schaffrath. The ambivalence of the barley Mlo locus: Mutations conferring resistance against powdery mildew (Blumeria graminis $\mathrm{f}$. sp. hordei) enhance susceptibility to the rice blast fungus Magnaporthe grisea. Molecular Plant-Microbe Interactions, 12(6):508-514, 1999. ISSN 0894-0282.

11. Katrin Gruner, Tatyana Zeier, Christina Aretz, and Jürgen Zeier. A critical role for Arabidopsis MILDEW RESISTANCE LOCUS O2 in systemic acquired resistance. The Plant Journal, 94(6):1064-1082, jun 2018. ISSN 09607412. doi: 10.1111/tpj.13920.

12. Fuqiang Cui, Hongpo Wu, Omid Safronov, Panpan Zhang, Rajeev Kumar, Hannes Kollist, Jarkko Salojärvi, Ralph Panstruga, and Kirk Overmyer. Arabidopsis MLO2 is a negative regulator of sensitivity to extracellular reactive oxygen species. Plant, Cell \& Environment, 41(4):782-796, apr 2018. ISSN 01407791. doi: 10.1111/pce.13144.

13. Alessandra Devoto, Pietro Piffanelli, I. Nilsson, E. Wallin, Ralph Panstruga, G. von Heijne, and Paul Schulze-Lefert. Topology, subcellular localization, and sequence diversity of the
Mlo family in plants. The Journal of Biological Chemistry, 274(49):34993-35004, 1999. ISSN 0021-9258. doi: 10.1074/jbc.274.49.34993.

14. Alessandra Devoto, H. Andreas Hartmann, Pietro Piffanelli, Candace Elliott, Carl Simmons, Graziana Taramino, Chern-Sing Goh, Fred E. Cohen, Brent C. Emerson, Paul SchulzeLefert, and Ralph Panstruga. Molecular phylogeny and evolution of the plant-specific seventransmembrane MLO family. Journal of Molecular Evolution, 56(1):77-88, 2003. ISSN 0022-2844. doi: 10.1007/s00239-002-2382-5.

15. Min Chul Kim, Ralph Panstruga, Candace Elliott, Judith Müller, Alessandra Devoto, Hae W. Yoon, Hyeong C. Park, Moo Je Cho, and Paul Schulze-Lefert. Calmodulin interacts with MLO protein to regulate defence against mildew in barley. Nature, 416(6879):447-451, 2002. ISSN 0028-0836. doi: 10.1038/416447a.

16. Candace Elliott, Judith Müller, Marco Miklis, Riyaz A. Bhat, Paul Schulze-Lefert, and Ralph Panstruga. Conserved extracellular cysteine residues and cytoplasmic loop-loop interplay are required for functionality of the heptahelical MLO protein. The Biochemical Journal, 385 (Pt 1):243-254, 2005. ISSN 1470-8728. doi: 10.1042/BJ20040993.

17. Ralph Panstruga. Serpentine plant MLO proteins as entry portals for powdery mildew fungi. Biochemical Society Transactions, 33(Pt 2):389-392, 2005. ISSN 0300-5127. doi: 10.1042/ BST0330389.

18. Michela Appiano, Domenico Catalano, Miguel Santillán Martínez, Concetta Lotti, Zheng Zheng, Richard G. F. Visser, Luigi Ricciardi, Yuling Bai, and Stefano Pavan. Monocot and dicot MLO powdery mildew susceptibility factors are functionally conserved in spite of the evolution of class-specific molecular features. BMC Plant Biology, 15:257, 2015. ISSN 1471-2229. doi: 10.1186/s12870-015-0639-6.

19. Stefan Kusch, Lina Pesch, and Ralph Panstruga. Comprehensive phylogenetic analysis sheds light on the diversity and origin of the MLO family of integral membrane proteins. Genome Biology and Evolution, 8(3):878-895, 2016. ISSN 1759-6653. doi: 10.1093/gbe/ evw036.

20. Johanna Acevedo-Garcia, Stefan Kusch, and Ralph Panstruga. Magical mystery tour: MLO proteins in plant immunity and beyond. New Phytologist, 204(2):273-281, 2014. ISSN 0028646X. doi: 10.1111/nph.12889.

21. Michela Appiano, Stefano Pavan, Domenico Catalano, Zheng Zheng, Valentina Bracuto, Concetta Lotti, Richard G. F. Visser, Luigi Ricciardi, and Yuling Bai. Identification of candidate $M L O$ powdery mildew susceptibility genes in cultivated Solanaceae and functional characterization of tobacco NtMLO1. Transgenic Research, 24(5):847-858, 2015. ISSN 0962-8819. doi: 10.1007/s11248-015-9878-4

22. Zhongying Chen, H. Andreas Hartmann, Ming-Jing Wu, Erin J. Friedman, Jin-Gui Chen, Matthew Pulley, Paul Schulze-Lefert, Ralph Panstruga, and Alan M. Jones. Expression analysis of the AtMLO gene family encoding plant-specific seven-transmembrane domain proteins. Plant Molecular Biology, 60(4):583-597, 2006. ISSN 0167-4412. doi: 10.1007/ s11103-005-5082-x.

23. Przemyslaw Bidzinski, Sandra Noir, Shermineh Shahi, Anja Reinstädler, Dominika Marta Gratkowska, and Ralph Panstruga. Physiological characterization and genetic modifiers of aberrant root thigmomorphogenesis in mutants of Arabidopsis thaliana MILDEW LOCUS O genes. Plant, Cell \& Environment, 37(12):2738-2753, 2014. ISSN 1365-3040. doi: 10.1111 pce. 12353 .

24. Zhongying Chen, Sandra Noir, Mark Kwaaitaal, H. Andreas Hartmann, Ming-Jing Wu, Yashwanti Mudgil, Poornima Sukumar, Gloria Muday, Ralph Panstruga, and Alan M. Jones. Two seven-transmembrane domain MILDEW RESISTANCE LOCUS O proteins cofunction in Arabidopsis root thigmomorphogenesis. The Plant Cell, 21(7):1972-1991, 2009. ISSN 1040-4651. doi: 10.1105/tpc.108.062653.

25. Sharon A. Kessler, Hiroko Shimosato-Asano, Nana F. Keinath, Samuel E. Wuest, Gwyneth Ingram, Ralph Panstruga, and Ueli Grossniklaus. Conserved molecular components for pollen tube reception and fungal invasion. Science (New York, N.Y.), 330(6006):968-971, 2010. ISSN 1095-9203. doi: 10.1126/science.1195211.

26. Daniel S. Jones and Sharon A. Kessler. Cell type-dependent localization of MLO proteins. Plant Signaling \& Behavio, page e1393135, oct 2017. ISSN 1559-2324. doi: 10.1080/ 15592324.2017.1393135.

27. Debbie Winter, Ben Vinegar, Hardeep Nahal, Ron Ammar, Greg V. Wilson, and Nicholas J. Provart. An "Electronic Fluorescent Pictograph" browser for exploring and analyzing largescale biological data sets. PLOS ONE, 2(8):e718, 2007. ISSN 1932-6203. doi: 10.1371/ journal.pone.0000718.

28. Yuichi Aoki, Yasunobu Okamura, Shu Tadaka, Kengo Kinoshita, and Takeshi Obayashi. ATTED-II in 2016: A plant coexpression database towards lineage-specific coexpression. Plant \& Cell Physiology, 57(1):e5, 2016. ISSN 0032-0781. doi: 10.1093/pcp/pcv165.

29. Michiel Van Bel, Tim Diels, Emmelien Vancaester, Lukasz Kreft, Alexander Botzki, Yves Van de Peer, Frederik Coppens, and Klaas Vandepoele. PLAZA 4.0: an integrative resource for functional, evolutionary and comparative plant genomics. Nucleic Acids Research, 46 (D1):D1190-D1196, jan 2018. ISSN 0305-1048. doi: 10.1093/nar/gkx1002.

30. Huaiyu Mi, Sagar Poudel, Anushya Muruganujan, John T. Casagrande, and Paul D. Thomas. PANTHER version 10: expanded protein families and functions, and analysis tools. Nucleic Acids Research, 44(D1):D336-342, 2016. ISSN 0305-1048. doi: 10.1093/nar/gkv1194.

31. Friederike Bernsdorff, Anne-Christin Döring, Katrin Gruner, Stefan Schuck, Andrea Bräutigam, and Jürgen Zeier. Pipecolic acid orchestrates plant systemic acquired resistance and defense priming via salicylic acid-dependent and -independent pathways. The Plant Cell, 28(1):102-129, dec 2016. ISSN 1040-4651. doi: 10.1105/tpc.15.00496.

32. Michael Hartmann, Tatyana Zeier, Friederike Bernsdorff, Vanessa Reichel-Deland, Denis Kim, Michele Hohmann, Nicola Scholten, Stefan Schuck, Andrea Bräutigam, Torsten Hölzel, Christian Ganter, and Jürgen Zeier. Flavin monooxygenase-generated N-hydroxypipecolic acid is a critical element of plant systemic immunity. Cell, 173(2):456-469.e16, apr 2018. ISSN 0092-8674. doi: 10.1016/J.CELL.2018.02.049.

33. Hana Návarová, Friederike Bernsdorff, Anne-Christin Döring, and Jürgen Zeier. Pipecolic acid, an endogenous mediator of defense amplification and priming, is a critical regulator of inducible plant immunity. The Plant Cell, 24(12):5123-5141, dec 2012. ISSN 1040-4651. doi: $10.1105 /$ tpc. 112.103564 .

34. Minghui Gao, Xia Wang, Dongmei Wang, Fang Xu, Xiaojun Ding, Zhibin Zhang, Dongling $\mathrm{Bi}, \mathrm{Yu} \mathrm{Ti}$ Cheng, She Chen, Xin Li, and Yuelin Zhang. Regulation of cell death and innate 
immunity by two receptor-like kinases in Arabidopsis. Cell Host \& Microbe, 6(1):34-44, jul 2009. ISSN 1931-3128. doi: 10.1016/J.CHOM.2009.05.019.

35. Steffen Rietz, Anika Stamm, Stefan Malonek, Stephan Wagner, Dieter Becker, Nieves Medina-Escobar, A. Corina Vlot, Bart J. Feys, Karsten Niefind, and Jane E. Parker. Different roles of Enhanced Disease Susceptibility1 (EDS1) bound to and dissociated from Phytoalexin Deficient4 (PAD4) in Arabidopsis immunity. New Phytologist, 191(1):107-119, 2011. ISSN 0028646X. doi: 10.1111/j.1469-8137.2011.03675.x.

36. Zheng Qing Fu, Shunping Yan, Abdelaty Saleh, Wei Wang, James Ruble, Nodoka Oka, Rajinikanth Mohan, Steven H. Spoel, Yasuomi Tada, Ning Zheng, and Xinnian Dong. NPR3 and NPR4 are receptors for the immune signal salicylic acid in plants. Nature, 486(7402): 228-232, jun 2012. ISSN 0028-0836. doi: 10.1038/nature11162.

37. Magali Moreau, Miaoying Tian, and Daniel F. Klessig. Salicylic acid binds NPR3 and NPR4 to regulate NPR1-dependent defense responses. Cell Research, 22(12):1631-1633, dec 2012. ISSN 1001-0602. doi: 10.1038/cr.2012.100.

38. Andrew F. Bent, B. Kunkel, D. Dahlbeck, K. L. Brown, Renate Schmidt, J. Giraudat, J. Leung, and Brian J. Staskawicz. RPS2 of Arabidopsis thaliana: a leucine-rich repeat class of plan disease resistance genes. Science (New York, N.Y.), 265(5180):1856-1860, sep 1994 ISSN 0036-8075. doi: 10.1126/science.8091210.

39. Michael Mindrinos, Fumiaki Katagiri, Guo-Liang Yu, and Frederick M. Ausubel. The A. thaliana disease resistance gene RPS2 encodes a protein containing a nucleotide-binding site and leucine-rich repeats. Cell, 78(6):1089-1099, sep 1994. ISSN 00928674. doi: 10.1016/0092-8674(94)90282-8.

40. Sébastien Besseau, Jing Li, and E. Tapio Palva. WRKY54 and WRKY70 co-operate as negative regulators of leaf senescence in Arabidopsis thaliana. Journal of Experimental Botany, 63(7):2667-2679, apr 2012. ISSN 1460-2431. doi: 10.1093/jxb/err450.

41. Yanru Hu, Quyan Dong, and Diqiu Yu. Arabidopsis WRKY46 coordinates with WRKY70 and WRKY53 in basal resistance against pathogen Pseudomonas syringae. Plant Science, 185-186:288-297, apr 2012. ISSN 0168-9452. doi: 10.1016/J.PLANTSCI.2011.12.003.

42. Jing Li, Günter Brader, Tarja Kariola, and E. Tapio Palva. WRKY70 modulates the selection of signaling pathways in plant defense. The Plant Journal, 46(3):477-491, may 2006. ISSN 09607412. doi: 10.1111/j.1365-313X.2006.02712.x.

43. Reinhard Hehl, Leo Norval, Artyom Romanov, and Lorenz Bülow. Boosting AthaMap database content with data from protein binding microarrays. Plant and Cell Physiology, 57(1):e4, jan 2016. ISSN 0032-0781. doi: 10.1093/pcp/pcv156.

44. Nils Ole Steffens, Claudia Galuschka, Martin Schindler, Lorenz Bülow, and Reinhard Hehl. AthaMap: an online resource for in silico transcription factor binding sites in the Arabidopsis thaliana genome. Nucleic Acids Research, 32(suppl_1):D368-D372, jan 2004. ISSN 1362 4962. doi: 10.1093/nar/gkh017.

45. Timothy L. Bailey, James Johnson, Charles E. Grant, and William Stafford Noble. The MEME Suite. Nucleic Acids Research, 43(W1):W39-49, 2015. ISSN 0305-1048. doi: 10.1093/nar/gkv416.

46. Volker Lipka, Jan Dittgen, Paweł Bednarek, Riyaz A. Bhat, Marcel Wiermer, Mónica Stein, Jörn Landtag, Wolfgang Brandt, Sabine Rosahl, Dierk Scheel, Francisco Llorente, Antonio Molina, Jane E. Parker, Shauna C. Somerville, and Paul Schulze-Lefert. Pre- and postinvasion defenses both contribute to nonhost resistance in Arabidopsis. Science (New York, N.Y.), 310(5751):1180-1183, 2005. ISSN 1095-9203. doi: 10.1126/science.1119409.

47. Eric B. Holub, Jim L. Beynon, and lan R. Crute. Phenotypic and genotypic characterization of interactions between isolates of Peronospora parasitica and accessions of Arabidopsis thaliana. Molecular Plant-Microbe Interactions, 7:223-239, 1994. ISSN 0894-0282.

48. Michael Bartsch, Enrico Gobbato, Paweł Bednarek, Svenja Debey, Joachim L. Schultze, Jaqueline Bautor, and Jane E. Parker. Salicylic acid-independent ENHANCED DISEASE SUSCEPTIBILITY1 signaling in Arabidopsis immunity and cell death is regulated by the monooxygenase FMO1 and the Nudix hydrolase NUDT7. The Plant Cell, 18(4):1038-1051, 2006. ISSN 1040-4651. doi: 10.1105/tpc.105.039982.

49. Adam J. Bogdanove, Steven V. Beer, Ulla Bonas, Christian A. Boucher, Alan Collmer, David L. Coplin, Guy R. Cornelis, Hsiou-Chen Huang, Steven W. Hutcheson, Nickolas J. Panopoulos, and Frederique van Gijsegem. Unified nomenclature for broadly conserved $h r p$ genes of phytopathogenic bacteria. Molecular Microbiology, 20(3):681-683, 1996. ISSN 0950-382X. doi: 10.1046/j.1365-2958.1996.5731077.x.

50. Wen-Ling Deng, Gail Preston, Alan Collmer, Chun-Jung Chang, and Hsiou-Chen Huang Characterization of the $h r p C$ and $h r p R S$ operons of Pseudomonas syringae pathovars sy ringae, tomato, and glycinea and analysis of the ability of $h r p F, h r p G, h r c C, h r p T$, and $h r p V$ mu. Journal of Bacteriology, 180(17):4523-4531, 1998. ISSN 0021-9193.

51. Jane E. Parker, Eric B. Holub, Louise N. Frost, Anders Falk, Nick D. Gunn, and Michael J. Daniels. Characterization of eds1, a mutation in Arabidopsis suppressing resistance to Peronospora parasitica specified by several different RPP genes. The Plant Cell, 8(11): 2033-2046, 1996. ISSN 1040-4651. doi: 10.1105/tpc.8.11.2033.

52. Jun Fan, Casey Crooks, and Chris Lamb. High-throughput quantitative luminescence assay of the growth in planta of Pseudomonas syringae chromosomally tagged with Photorhabdus luminescens luxCDABE. The Plant Journal, 53(2):393-399, sep 2007. ISSN 09607412. doi: 10.1111/j.1365-313X.2007.03303.x.

53. Anne E. Carpenter, Thouis R. Jones, Michael R. Lamprecht, Colin Clarke, In Han Kang Ola Friman, David A. Guertin, Joo Han Chang, Robert A. Lindquist, Jason Moffat, Polina Golland, and David M. Sabatini. CellProfiler: image analysis software for identifying and quantifying cell phenotypes. Genome Biology, 7(10):R100, 2006. ISSN 1465-6914.

54. Andrew K. Jacobs, Volker Lipka, Rachel A. Burton, Ralph Panstruga, Nicolai Strizhov, Pau Schulze-Lefert, and Geoffrey B. Fincher. An Arabidopsis callose synthase, GSL5, is required for wound and papillary callose formation. The Plant Cell, 15(11):2503-2513, oct 2003. ISSN 1040-4651. doi: $10.1105 /$ tpc.016097.

55. Marc T. Nishimura, Mónica Stein, Bi-Huei Hou, John P. Vogel, Herb Edwards, and Shauna C Somerville. Loss of a callose synthase results in salicylic acid-dependent disease resistance. Science (New York, N.Y.), 301(5635):969-972, 2003. ISSN 1095-9203.

56. Justine Lorek, Thomas Griebel, Alan M. Jones, Hannah Kuhn, and Ralph Panstruga. The role of Arabidopsis heterotrimeric G-protein subunits in MLO2 function and MAMP-triggered immunity. Molecular Plant-Microbe Interactions, 26(9):991-1003, 2013. ISSN 0894-0282. doi: 10.1094/MPMI-03-13-0077-R.
57. Alan B. Rose, Tali Elfersi, Genis Parra, and lan Korf. Promoter-proximal introns in Arabidopsis thaliana are enriched in dispersed signals that elevate gene expression. The Plant Cell, 20(3):543-551, mar 2008. ISSN 1040-4651. doi: 10.1105/tpc.107.057190.

58. Bjoern O. Hansen, Neha Vaid, Magdalena Musialak-Lange, Marcin Janowski, and Marek Mutwil. Elucidating gene function and function evolution through comparison of coexpression networks of plants. Frontiers in Plant Science, 5:394, aug 2014. ISSN 1664462X. doi: 10.3389/fpls.2014.00394.

59. Matthew E. Humphry, Paweł Bednarek, Birgit Kemmerling, Serry Koh, Mónica Stein, UIrike Göbel, Kurt Stüber, Mariola Piślewska-Bednarek, Ann Loraine, Paul Schulze-Lefert, Shauna C. Somerville, and Ralph Panstruga. A regulon conserved in monocot and dicot plants defines a functional module in antifungal plant immunity. Proceedings of the National Academy of Sciences of the United States of America, 107(50):21896-21901, 2010. ISSN 0027-8424. doi: 10.1073/pnas.1003619107.

60. Moritz Schön, Armin Töller, Celia Diezel, Charlotte Roth, Lore Westphal, Marcel Wiermer, and Imre E. Somssich. Analyses of wrky18 wrky40 plants reveal critical roles of SA/EDS1 signaling and indole-glucosinolate biosynthesis for Golovinomyces orontii resistance and a loss-of resistance towards Pseudomonas syringae pv. tomato AvrRPS4 Molecular Plant-Microbe Interactions, 26(7):758-767, jul 2013. ISSN 0894-0282. doi: 10.1094/MPMI-11-12-0265-R.

61. Kang-Chang Kim, Zhibing Lai, Baofeng Fan, and Zhixiang Chen. Arabidopsis WRKY38 and WRKY62 transcription factors interact with Histone Deacetylase 19 in basal defense. The Plant Cell, 20(9):2357-2371, sep 2008. ISSN 1040-4651. doi: 10.1105/tpc.107.055566.

62. Riyaz A. Bhat, Marco Miklis, Elmon Schmelzer, Paul Schulze-Lefert, and Ralph Panstruga. Recruitment and interaction dynamics of plant penetration resistance components in a plasma membrane microdomain. Proceedings of the National Academy of Sciences of the United States of America, 102(8):3135-3140, 2005. ISSN 0027-8424. doi: 10.1073/ pnas.0500012102.

63. William Underwood and Shauna C. Somerville. Perception of conserved pathogen elicitors at the plasma membrane leads to relocalization of the Arabidopsis PEN3 transporter. Proceedings of the National Academy of Sciences of the United States of America, 110(30): 12492-12497, 2013. ISSN 0027-8424 doi: 10.1073/pnas.1218701110.

64. Dorit Meyer, Simone Pajonk, Cristina O. Micali, Richard J. O'Connell, and Paul SchulzeLefert. Extracellular transport and integration of plant secretory proteins into pathogeninduced cell wall compartments. The Plant Journal, 57(6):986-999, mar 2009. ISSN 09607412. doi: 10.1111/j.1365-313X.2008.03743.x.

65. Johanna Acevedo-Garcia, David Spencer, Hannah Thieron, Anja Reinstädler, Kim E. Hammond-Kosack, Andrew L. Phillips, and Ralph Panstruga. mlo-based powdery mildew resistance in hexaploid bread wheat generated by a non-transgenic TILLING approach. Plant Biotechnology Journal, 15(3):367-378, 2017. ISSN 1467-7652. doi: 10.1111/pbi. 12631 .

66. Philip Carella, Daniel C. Wilson, and Robin K. Cameron. Some things get better with age: differences in salicylic acid accumulation and defense signaling in young and mature Arabidopsis. Frontiers in Plant Science, 5:775, jan 2015. ISSN 1664-462X. doi: 10.3389/fpls.2014.00775

67. Julianne V. Kus, Kasia Zaton, Raani Sarkar, and Robin K. Cameron. Age-related resistance in Arabidopsis is a developmentally regulated defense response to Pseudomonas syringae. The Plant Cell, 14(2):479-490, feb 2002. ISSN 1040-4651. doi: 10.1105/TPC.010481.

68. C. Rusterucci, Z. Zhao, K. Haines, D. Mellersh, M. Neumann, and R. K. Cameron. Agerelated resistance to Pseudomonas syringae pv. tomato is associated with the transition to flowering in Arabidopsis and is effective against Peronospora parasitica. Physiological and Molecular Plant Pathology, 66(6):222-231, jun 2005. ISSN 0885-5765. doi: 10.1016/J. PMPP.2005.08.004.

69. Daniel S. Jones, Jing Yuan, Benjamin E. Smith, Andrew C. Willoughby, Emily L. Kumimoto, and Sharon A. Kessler. MILDEW RESISTANCE LOCUS O function in pollen tube reception is linked to its oligomerization and subcellular distribution. Plant Physiology, 175(1):172185, sep 2017. ISSN 1532-2548. doi: 10.1104/pp.17.00523.

70. José M. Alonso, Anna N. Stepanova, Thomas J. Leisse, Christopher J. Kim, Huaming Chen, Paul Shinn, Denise K. Stevenson, Justin Zimmerman, Pascual Barajas, Rosa Cheuk, Carmelita Gadrinab, Collen Heller, Albert Jeske, Eric Koesema, Cristina C. Meyers, Holly Parker, Lance Prednis, Yasser Ansari, Nathan Choy, Hashim Deen, Michae Geralt, Nisha Hazari, Emily Hom, Meagan Karnes, Celene Mulholland, Ral Ndubaku, lan Schmidt, Plinio Guzman, Laura Aguilar-Henonin, Markus Schmid, Detlef Weigel, David E. Carter, Trudy Marchand, Eddy Risseeuw, Debra Brogden, Albana Zeko, William L. Crosby, Charles C. Berry, and Joseph R. Ecker. Genome-wide insertional mutagenesis of Arabidopsis thaliana. Science (New York, N.Y.), 301(5633):653-657, 2003. ISSN 1095-9203. doi: 10.1126/science. 1086391.

71. Vitali Matyash, Gerhard Liebisch, Teymuras V. Kurzchalia, Andrej Shevchenko, and Dominik Schwudke. Lipid extraction by methyl-tert-butyl ether for high-throughput lipidomics. Journal of Lipid Research, 49(5):1137-1146, may 2008. ISSN 0022-2275. doi: 10.1194/jlr. D700041-JLR200.

72. Gerd Ulrich Balcke, Vinzenz Handrick, Nick Bergau, Mandy Fichtner, Anja Henning, Hagen Stellmach, Alain Tissier, Bettina Hause, and Andrej Frolov. An UPLC-MS/MS method for highly sensitive high-throughput analysis of phytohormones in plant tissues. Plant Methods, 8(1):47, nov 2012. ISSN 1746-4811. doi: 10.1186/1746-4811-8-47.

73. Tim Iven, Stefanie König, Seema Singh, Susanna A. Braus-Stromeyer, Matthias Bischoff, Lutz F. Tietze, Gerhard H. Braus, Volker Lipka, Ivo Feussner, and Wolfgang Dröge-Laser. Transcriptional activation and production of tryptophan-derived secondary metabolites in Arabidopsis roots contributes to the defense against the fungal vascular pathogen Verticillium longisporum. Molecular Plant, 5(6):1389-1402, nov 2012. ISSN 1674-2052. doi: 10.1093/MP/SSS044.

74. Hadley Wickham. ggplot2: Elegant Graphics for Data Analysis. Springer-Verlag., New York, 1 edition, 2009. ISBN 978-0-387-98141-3. doi: 10.1007/978-0-387-98141-3.

75. Michael J. Crawley. Statistics: An introduction using R. John Wiley \& Sons, Ltd, Imperia College London, UK, second edi edition, 2015. ISBN 978-1-118-94109-6. 


\section{Supplementary data}

Table S1. AtMLO3 is upregulated in systemic leaves of Arabidopsis accession Col-0 during SAR and in response to application of pipecolic acid (Pip). The table was compiled on the basis of data from Bernsdorff et al. (2016) and Hartmann et al. (2018). Expression is shown as reads per million (RPM), asterisks indicate significant expression differences.

\begin{tabular}{|c|c|c|c|c|c|c|c|c|c|c|}
\hline & & Col-0 & & & fmol & & & Col-0 & & \\
\hline Name & AGI code & $\mathrm{H}_{2} \mathrm{O}$ & Pip $^{b}$ & $\log \mathrm{FC}^{\mathrm{c}}$ & $\mathrm{H}_{2} \mathrm{O}$ & Pip $^{b}$ & $\log F C^{c}$ & Mock & Psm & $\log F C^{c}$ \\
\hline AtMLOI & At4g02600 & 24.1 & 32.2 & 0.4 & 28.0 & 29.1 & 0.1 & 32.7 & 39.3 & 0.3 \\
\hline AtMLO2 & Atlg11310 & 55.6 & 145.1 & $1.4 * *$ & 48.3 & 52.7 & 0.1 & 78.6 & 515 & $2.7 * *$ \\
\hline AtMLO3 & At3g45290 & 5.1 & 16.5 & $1.5 * *$ & 5.2 & 5.2 & 0.0 & 10.3 & 57.5 & $2.4 * *$ \\
\hline AtMLO4 & At1g11000 & 6.8 & 9.3 & 0.4 & 7.7 & 8.3 & 0.1 & 6.2 & 8.2 & 0.4 \\
\hline AtMLO5 & At2g33670 & 0.0 & 0.0 & 0.0 & 0.0 & 0.0 & 0.0 & 0.0 & 0.0 & 0.0 \\
\hline AtMLO6 & At1g61560 & 0.9 & 2.8 & 1.0 & 0.8 & 0.7 & 0.0 & 1.1 & 19.2 & $3.3^{* *}$ \\
\hline AtMLO7 & At2g17430 & 1.3 & 2.4 & 0.5 & 1.3 & 1.7 & 0.2 & 1.2 & 2.1 & 0.5 \\
\hline AtMLO8 & At2g17480 & 13.3 & 14.8 & 0.1 & 13.4 & 15.6 & 0.2 & 20.4 & 20.2 & 0.0 \\
\hline AtMLO9 & At1g42560 & 0.1 & 0.1 & 0.0 & 0.2 & 0.2 & -0.1 & 0.0 & 0.0 & 0.0 \\
\hline AtMLO10 & At5g65970 & 4.0 & 5.2 & 0.3 & 3.3 & 3.7 & 0.1 & 2.9 & 4.0 & 0.4 \\
\hline AtMLO11 & At5g53760 & 15.0 & 13.5 & -0.1 & 15.2 & 15.9 & 0.1 & 14.2 & 6.7 & $-1.0 *$ \\
\hline AtMLO12 & At2g39200 & 0.3 & 0.4 & 0.1 & 0.5 & 0.3 & -0.2 & 0.6 & 5.7 & $2.1 * *$ \\
\hline AtMLO13 & At4g24250 & 0.9 & 1.4 & 0.4 & 0.8 & 1.1 & 0.2 & 0.5 & 0.5 & 0.0 \\
\hline AtMLO14 & At1g26700 & 0.0 & 0.0 & 0.0 & 0.0 & 0.0 & 0.0 & 0.0 & 0.0 & 0.0 \\
\hline AtMLO15 & At2g44110 & 0.0 & 0.0 & 0.0 & 0.0 & 0.0 & 0.0 & 0.0 & 0.0 & 0.0 \\
\hline
\end{tabular}

a fmol, Arabidopsis mutant of FLAVIN MONOOXYGENASE1 (FMOI)

${ }^{\mathrm{b}}$ Pip, Pipecolic acid applied by watering

${ }^{\mathrm{c}} \log \mathrm{FC}, \log _{2}$ fold change 
bioRxiv preprint doi: https://doi.org/10.1101/558122; this version posted February 22, 2019. The copyright holder for this preprint (which was not certified by peer review) is the author/funder. All rights reserved. No reuse allowed without permission.

Table S2. Summary of significant differences in phytohormone compound compositions in mlo2 and mlo3 compared to Col-0.

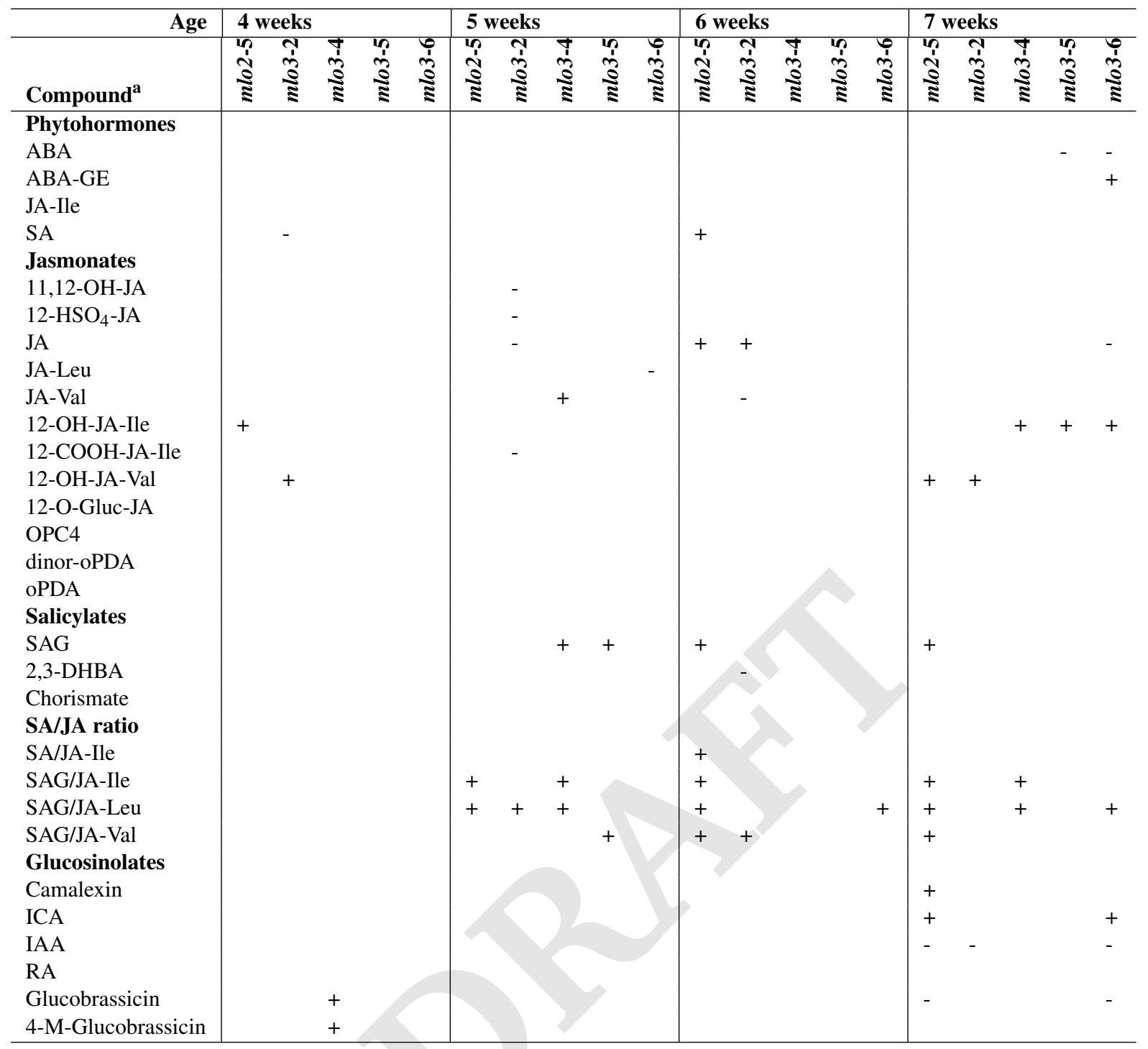

${ }^{a}$ The abbreviations are as follows: ABA, abscisic acid; ABA-GE, abscisic acid-glucose ester (inactive); JA, jasmonic acid; SA, salicylic acid; OH-JA, Hydroxyjasmonic acid; $\mathrm{HSO}_{4}-\mathrm{JA}$, hydroxyjasmonic acid sulfate; JA-Ile, JA-isoleucine; JA-Leu, JA-leucine; JA-Val, JA-valine; COOH-JA-Ile, dicarboxy-JA-Ile; O-Gluc-JA, O-glucose-JA; OPC4, 3-oxo-2-(2-(Z)-pentenyl)cyclopentane-1-butanoic acid; oPDA, 12-oxo-phytodienoic acid; SAG, SA-glucoside; 2,3-DHBA, 2,3-Dihydroxybenzoic acid; ICA, indole-carboxylic acid; IAA, indole-3-acetic acid; RA, raphanusamic acid. 
Table S3. Mass transitions and corresponding conditions for determination of the phytohormones.

\begin{tabular}{llllll}
\hline \multicolumn{2}{l}{ MRM transitions } & & & & \\
\hline $\mathbf{Q 1}^{\mathbf{a}}$ & $\mathbf{Q 3}^{\mathbf{a}}$ & Analyte & $\mathbf{D P}^{\mathbf{b}}$ & $\mathbf{E P}^{\mathbf{c}}$ & $\mathbf{C E}^{\mathbf{d}}$ \\
\hline 137 & 93 & SA & -25 & -6 & -20 \\
141 & 97 & D4-SA & -25 & -6 & -22 \\
153 & 109 & 2,3-DHBA & -25 & -9 & -18 \\
160 & 116 & ICA & -40 & -6.5 & -22 \\
162 & 58 & RA & -15 & -6 & -14 \\
174 & 130 & IAA & -35 & -9 & -14 \\
179 & 135 & D5-IAA & -35 & -9 & -14 \\
207 & 137 & Chorismat-H 2 O & -20 & -9 & -16 \\
209 & 59 & JA & -30 & -4.5 & -24 \\
214 & 62 & D5-JA & -35 & -8.5 & -24 \\
225 & 59 & 11,12-OH-JA & -35 & -9 & -28 \\
237 & 165 & OPC4 & -45 & -6 & -24 \\
263 & 153 & ABA & -35 & -4 & -14 \\
263 & 165 & dinor-oPDA & -40 & -5 & -20 \\
293 & 179 & D6-ABA & -80 & -10 & -42 \\
296 & 170.2 & D5-oPDA & -65 & -4 & -28 \\
299 & 137 & SAG & -30 & -4 & -18 \\
305 & 97 & 12-HSO $4-J A$ & -30 & -4 & -32 \\
308 & 116 & JA-Val & -45 & -5 & -28 \\
322 & 130 & JA-Ile/Leu & -45 & -5 & -28 \\
325 & 133 & D4-JA-Leu & -80 & -4 & -30 \\
324 & 116 & 12OH-JA-Val & -45 & -10 & -30 \\
338 & 130 & 12OH-JA-Ile & -45 & -10 & -30 \\
352 & 130 & 12COOH-JA-Ile & -45 & -10 & -30 \\
387 & 59 & 12-O-Gluc-JA & -85 & -9 & -59 \\
425 & 263 & ABA-GE & -30 & -10 & -16 \\
447 & 97 & Glucobrassicin & -45 & -7 & -40 \\
477 & 97 & 4-M-glucobrassicin & -55 & -5 & -38 \\
\hline & & & & & \\
\hline
\end{tabular}

${ }^{\text {a }} \mathrm{Q} 1$, mass of precursor ion; Q3, mass of product after fragmentation

${ }^{\mathrm{b}} \mathrm{DP}$, declustering potential

c EP, entrance potential

${ }^{\mathrm{d}} \mathrm{CE}$, collision energy 
Supplementary Files. List of supplementary files.

Supplementary File 1. Co-expressed genes of AtMLO1, AtMLO2, and AtMLO3 after ATTED-II release 2017.12.14.

Supplementary File 2. GO terms of the 79 genes from the common co-expression network of AtMLO2 and AtMLO3.

Supplementary File 3. Cis-regulatory elements of the 79 genes from the common co-expression network of AtMLO2 and $A t M L O 3$ predicted by AthaMap.

Supplementary File 4. Cis-regulatory elements of the 79 genes from the common co-expression network of AtMLO2 and AtMLO3 predicted by MEME v5.0.4. 


\section{AtMLO3 genomic (3827 bp)}

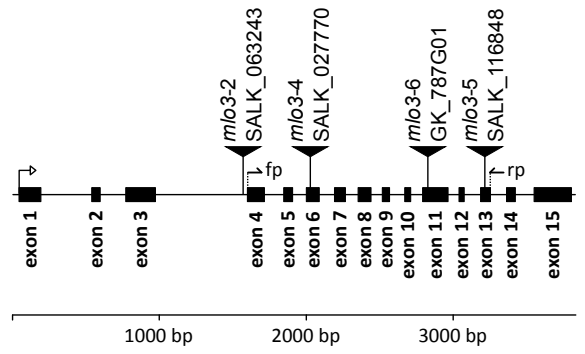

B
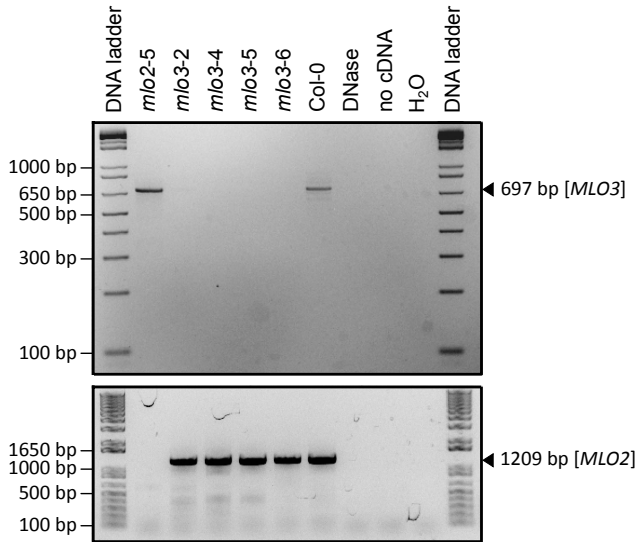

challenged cell

P35S::AtMLO3-YFP unchallenged cell

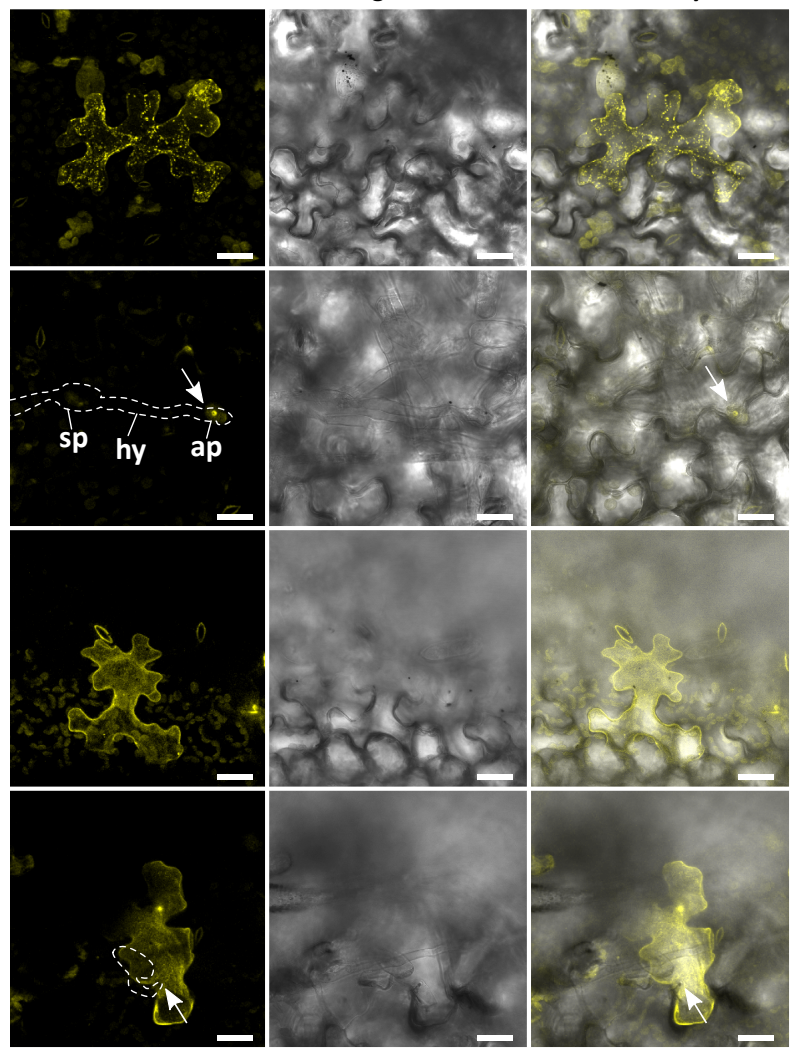



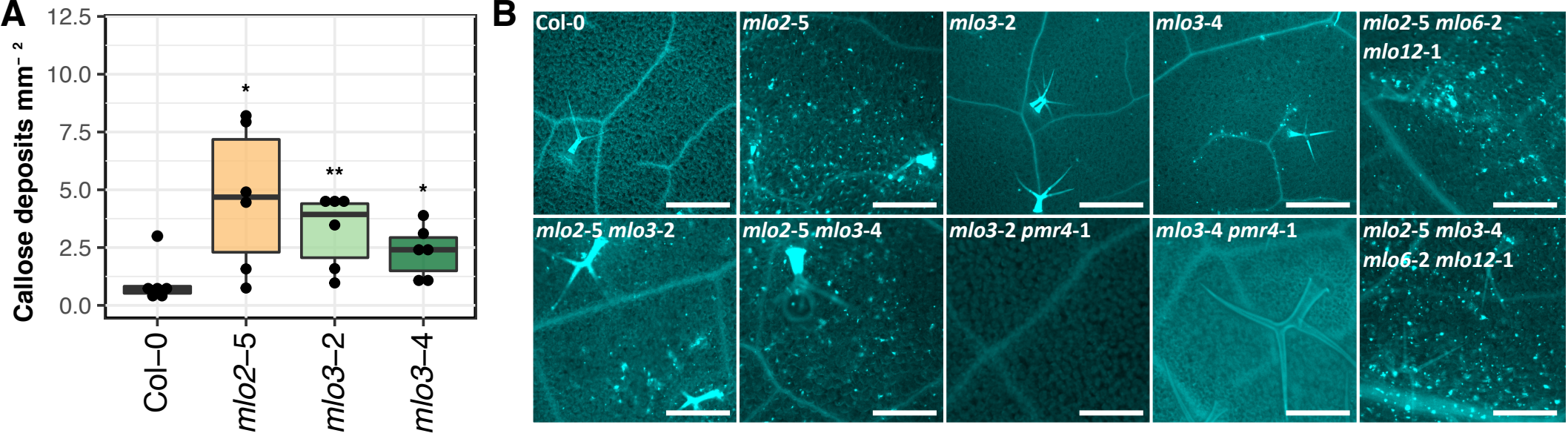

C

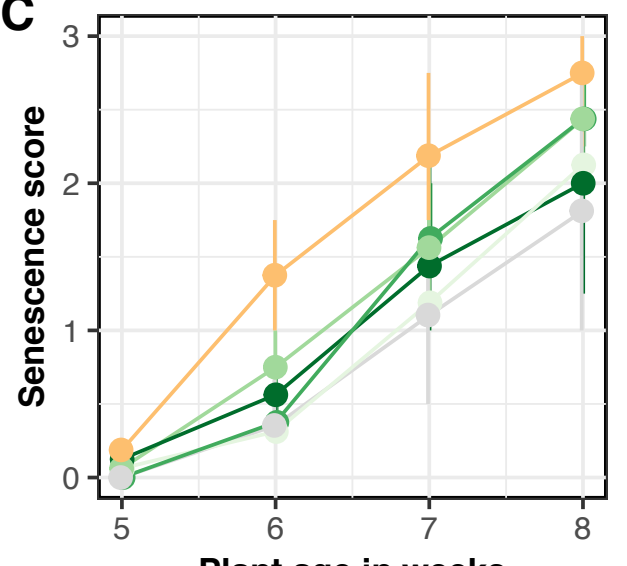

Plant age in weeks

\section{Genotype}

- Col-0

- mlo2-5

- mlo3-2

- mlo3-4 mlo3-5

- mlo3-6
D

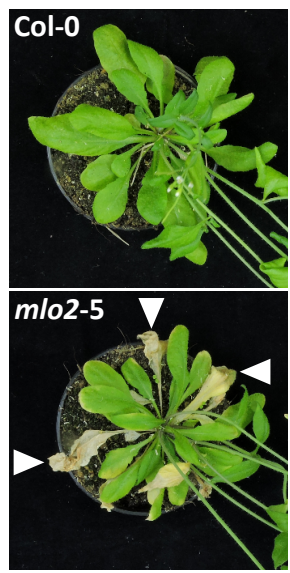

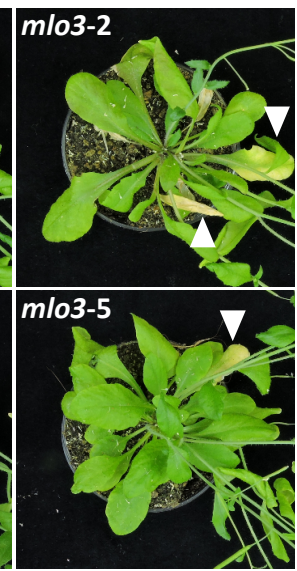

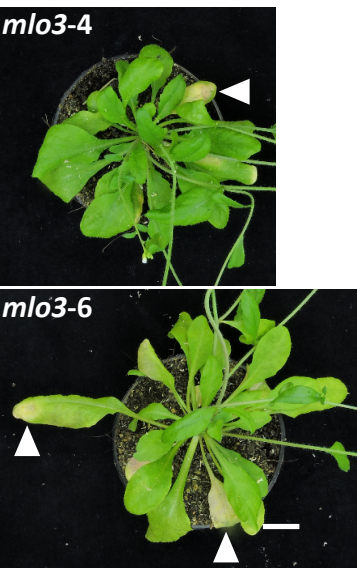




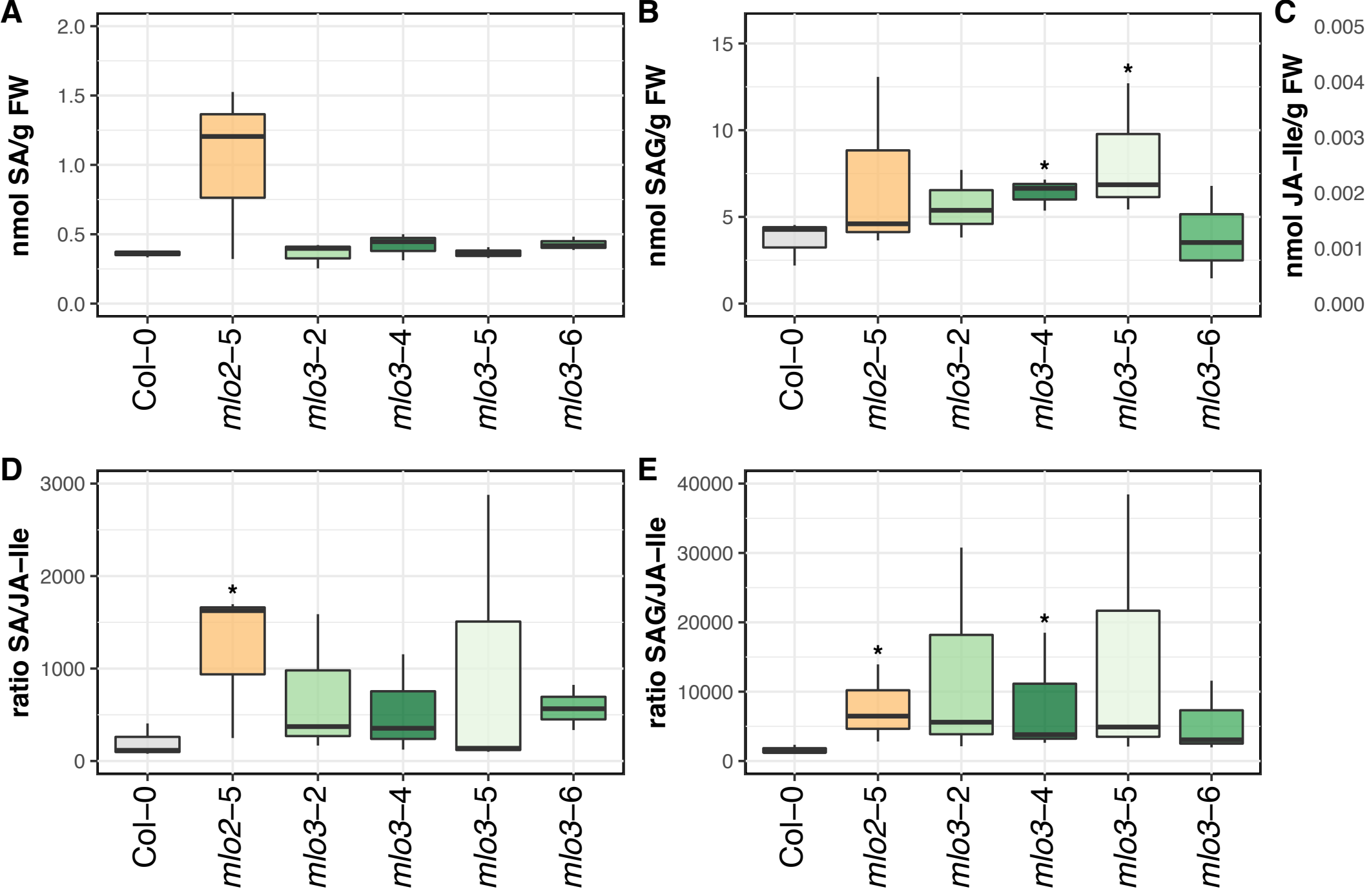

